\section{Post-translational polymodification of B1-tubulin regulates motor protein localization in platelet production and function}

\author{
Abdullah 0. Khan, ${ }^{1}$ Alexandre Slater, ${ }^{1}$ Annabel Maclachlan, ${ }^{1}$ Phillip L.R. \\ Nicolson, ${ }^{1}$ Jeremy A. Pike, ${ }^{1,2}$ Jasmeet S. Reyat, ${ }^{1}$ Jack Yule, ${ }^{1,2}$ Rachel Stapley, ${ }^{1}$ \\ Julie Rayes, ${ }^{1}$ Steven G. Thomas, ${ }^{1,2}$ and Neil V. Morgan ${ }^{1}$ \\ ${ }^{1}$ Institute of Cardiovascular Sciences, College of Medical and Dental Sciences, \\ University of Birmingham, Edgbaston, Birmingham and ${ }^{2}$ The Centre of Membrane \\ Proteins and Receptors (COMPARE), University of Birmingham and University of \\ Nottingham, Midlands, UK
}

\section{ABSTRACT}

$\tau$ n specialized cells, the expression of specific tubulin isoforms and their subsequent post-translational modifications drive and coordinate unique morphologies and behaviors. The mechanisms by which $\beta 1$-tubulin, the platelet and megakaryocyte $(\mathrm{MK})$ lineage restricted tubulin isoform, drives platelet production and function remains poorly understood. We investigated the roles of two key post-translational tubulin polymodifications (polyglutamylation and polyglycylation) on these processes using a cohort of thrombocytopenic patients, human induced pluripotent stem cell derived $M K$, and healthy human donor platelets. We find distinct patterns of polymodification in $M K$ and platelets, mediated by the antagonistic activities of the cell specific expression of tubulin tyrosine ligase like enzymes and cytosolic carboxypeptidase enzymes. The resulting microtubule patterning spatially regulates motor proteins to drive proplatelet formation in megakaryocytes, and the cytoskeletal reorganization required for thrombus formation. This work is the first to show a reversible system of polymodification by which different cell specific functions are achieved.

\section{Introduction}

Microtubules are large, cytoskeletal filaments vital to a host of critical functions including cell division, signaling, cargo transport, motility, and function. ${ }^{1,2}$ The question of how ubiquitously expressed filaments can facilitate complex and highly unique behaviors (such as neurotransmitter release and retinal organization) has been addressed by the concept of the tubulin code. ${ }^{3,4}$ This paradigm accounts for the specialization of microtubules and their organization by describing a mechanism in which particular cells express lineage restricted isoforms of tubulin. These cell specific isoforms are then subject to a series of post-translational modifications (PTM) which alter the mechanical properties of microtubules, and their capacity to recruit accessory proteins (e.g., motor proteins). ${ }^{1,2,4,5}$

A host of PTM have been reported in a range of cell types, including tyrosination, acetylation, glutamylation, glycylation, and phosphorylation. The loss of specific tubulin PTM has been linked to disease and dysfunction in motile and non-motile cilia (including respiratory cilia, retinal cells), spermatogenesis, muscular disorders, and neurological development. ${ }^{1,46-11}$ Despite an increasing understanding of the importance of tubulin PTM in disease, the role of the tubulin code in the generation of blood platelets from their progenitors, megakaryocytes $(\mathrm{MK})$ remains poorly understood.

Platelets are the smallest component of peripheral blood, and circulate as anucleate cells with an archetypal discoid shape maintained by a microtubule marginal band..$^{12,13}$ Antagonistic motor proteins maintain the resting state of the marginal band, and during platelet activation a motor dependent mechanism results in sliding which extends the marginal band and causes the transition to a spherical shape. ${ }^{13-15}$

Conversely $\mathrm{MK}$ are the largest and rarest hematopoeitic cells of the bone marrow.
Haematologica 2022

Volume 107(1):243-259

\section{Correspondence:}

ABDULLAH O. KHAN

a.khan.4@bham.ac.uk

NEIL V. MORGAN

N.V.Morgan@bham.ac.uk.

Received: August 28, 2020.

Accepted: December 11, 2020.

Pre-published: December 17, 2020.

https://doi.org/10.3324/haematol.2020.270793

(C)2022 Ferrata Storti Foundation

Material published in Haematologica is covered by copyright. All rights are reserved to the Ferrata Storti Foundation. Use of published material is allowed under the following terms and conditions:

https://creativecommons.org/licenses/by-nc/4.0/legalcode. Copies of published material are allowed for personal or internal use. Sharing published material for non-commercial purposes is subject to the following conditions:

https://creativecommons.org/licenses/by-nc/4.0/legalcode, sect. 3. Reproducing and sharing published material for commercial purposes is not allowed without permission in writing from the publisher. 
These cells are characteristically large, polyploid cells with unique morphological structures (e.g., the invaginated membrane system [IMS]) required to facilitate the production of thousands of blood platelets and packaged within them the required pro-thrombotic factors. ${ }^{15,16} \mathrm{MK}$ form long, beaded extensions into the lumen of bone marrow sinusoids - where these proplatelet extensions then experience fission under the flow of sinusoidal blood vessels which results in the release of barbell shaped pre-platelets and platelets into the blood stream. ${ }^{16}$

Both $\mathrm{MK}$ and platelets express a lineage-restricted isoform of $\beta 1$-tubulin encoded by the gene TUBB1. ${ }^{17}$ In humans, TUBB1 mutations have been shown to result in impaired platelet production, with a resulting macrothrombocytopenia. ${ }^{18,19}$ More recently, a C-terminal truncation of $\beta 1$-tubulin has been shown to cause a macrothrombocytopenia, suggesting that C-terminal modifications may be drivers of protein function and causative of the disease phenotype observed. ${ }^{20}$

While the loss of $\beta 1$-tubulin is known to result in macrothrombocytopenia, the mechanisms by which this isoform of tubulin effects the dramatically different cytoskeletal behaviors of platelets and MK remains poorly understood. In the context of the tubulin code, MK and platelets present a particularly interesting model. Both cells express $\beta 1$-tubulin, but undergo markedly different cytoskeletal changes. To date, acetylation and tyrosination have been the PTM primarily reported in MK and platelets, however neither modification is specific to the C-terminal tail encoded by TUBB1. Fiore et al. showed that a C-terminal truncation of $\beta 1$-tubulin phenocopies the complete loss of the protein. ${ }^{20} \mathrm{We}$, therefore, hypothesize that PTM specific to the C-terminus of TUBB1 are required for the complex morphological rearrangements required for both $\mathrm{MK}$ and platelet function.

The C-terminal tail of $\beta 1$ tubulin is particularly rich in glutamate residues which are often targeted for two key PTM implicated in human disease. Polyglutamylation and polyglycylation are PTM which target glutamate residues on both tubulin subunits ( $\alpha$ and $\beta$ ) and result in the addition of glutamate or glycine residues respectively.,1,21 As both PTM target the same residue they are together referred to as polymodification. Polymodification has been observed in microtubules in centrioles, axenomes, neuronal outgrowths, and mitotic spindles ${ }^{1,2}$

To date polyglycylation has not been reported in MK or platelets. Recently Van Dijk et al. reported on the polyglutamylation of $\beta 1$ tubulin downstream of the integrin $\alpha 2, \beta_{\text {s }}$ in a $\mathrm{CHO}$ cell line engineered to express TUBB1, murine $\mathrm{MK}$, and platelets spread on fibrinogen. ${ }^{22}$ In our work, we report the effects of the loss of the C-terminus of $\beta 1$-tubulin in patients with rare TUBB1 variants linked to the low platelet counts. Using induced pluripotent stem cell (iPSC) derived MK and CRISPR knockout (KO), we report a mechanism of polymodification which distinctly patterns $\beta 1$ tubulin in MK and platelets to spatially coordinate key motors. We describe the graded, cell specific expression of enzymes which mediate this, and finally report a novel gene, TTLL10, which is associated with excessive bleeding.

\section{Methods}

\section{Study approval}

Whole blood was obtained for each experiment from healthy volunteers under the University of Birmingham's ERN 11-0175 license 'The regulation of activation of platelets'. The Genotyping and Phenotyping of Platelets (GAPP) study was approved by the National Research Ethics Service Committee West Midlands - Edgbaston (REC reference 06/MRE07/36). Participants gave written informed consent in compliance with the Declaration of Helsinki. The GAPP study is included in the National Institute for Health Research Non-Malignant Hematology study portfolio (ID 9858), and registered at ISRCTN (http://www.isrctn.org) as ISRCTN77951167.

\section{Whole exome sequencing}

In order to identify the possible causative variants in these families we sequenced the whole exome of the affected individuals with the SureSelect human All Exon $50 \mathrm{Mb}$ kit (Agilent Technologies) and sequenced on the HiSeq 2500 (Illumina) with $100 \mathrm{bp}$ paired-end reads.

\section{Stem cell culture and induced pluripotent stem cell megakayocyte differentiation}

Gibco human episomal iPSC line was purchased from Thermo Scientific and cultured on Geltrex basement membrane in StemFlex medium (Thermo Scientific). iPSC differentiation to mature, proplatelet forming $\mathrm{MK}$ was performed using a protocol based on work published by Feng et al. ${ }^{23}$ The IDT Alt-R®RNP system was used to target and knock out TUBB1. iPSC transfection was performed using Lipofectamine Stem (Life Technologies) according to the manufacturer instructions.

\section{TUBB1 homology modeling}

Homology models of TUBB1 wild-type (WT) and variants were made using SWISS MODEL software, ${ }^{24-27}$ using the solved TUBB3 heterodimer as a template PDB: $5 \mathrm{IJO} 0^{28}$. TUBB 1 and TUBB3 share approximately $80 \%$ sequence identity, and the model created corresponds to residues $1-425$ of TUBB1. (Note that the Cterminal tail of TUBB1 is not presented in this model as there is no known or homologous structure available for this highly divergent sequence. The C-terminal portion of this model is used only as a means to visualize the effect of genetic variations on the C-terminal tail of TUBB1).

\section{Statistical analysis}

Statistical analysis was performed using GraphPad PRISM 7. Specifics of each test are detailed in the figure legends of the relevant figures. $P$-values below 0.05 were considered significant.

Detailed Materials and Methods can be seen in the Online Supplementary Appendix.

\section{Results}

\section{Identification and initial characterization of TUBB1 variants in patients with inherited thrombocytopenia and platelet dysfunction}

Using whole exome sequencing and Congenica Clinical genetic variant interpretation software ${ }^{29}$ of patients recruited to the GAPP study, two C-terminal TUBB1 variants were identified in unrelated families presenting with macrothrombocytopenia (Figure 1A; Online Supplementary Figure S1). Affected individuals in family A were found to be heterozygous for an arginine to tryptophan amino acid substitution (c.1075C > T, p.R359W) in TUBB1. Individuals in this family also carry a GFI1B variant (p.Cys168Phe). Variants in both genes have been linked to thrombocytopenia, however only individuals A:1 and A:3 (carrying 
TUBB1 variants), present with a macrothrombocytopenia (107 $\times 10^{9} / \mathrm{L}$ and $85 \times 10^{\circ} / \mathrm{L}$ respectively). Individual A:2 carries the GFI1B variant but is WT for TUBB1 and presents with a normal platelet count $\left(221 \times 10^{9} / \mathrm{L}\right)$. Individuals A:1 and A:3 also present with significantly higher immature platelet fractions (IPF) and mean platelet volumes (MPV) when compared to their TUBB1 WT relatives $(53.5 \%$ and $55.1 \%$ compared to $24.5 \%$, MPV for A:1 and A:3 too large for measurement). This variation in count, IPF and platelet morphology in individuals with the TUBB1 R359W variant may suggest that the $T U B B 1$ variant is linked to the macrothrombocytopenia observed.

Family/patient B was an elderly gentleman (now deceased) with a G insertion and subsequent frameshift truncation of the $\beta 1$-tubulin protein 19 amino acids from the site of insertion (c.1080insG, p.L361Afs*19). ${ }^{30,31}$ This patient had a severe thrombocytopenia with a platelet count of $11 \times 10^{9} / \mathrm{L}$ and an MPV above 13.4 (Figure 1B). At the time of study, IPF measurement was unavailable.

p.L361Afs*19 is absent in the latest version of the gnomad database (accessed October 2020), while p.R359W is a rare variant with a frequency of $6.78 \times 10^{-3}$ (gnomad accessed October 2020) with a significant pathogenicity prediction score of 25.4 using combined annotation dependent depletion (CADD), where a score of greater or equal to 20 indicates the $1 \%$ most deleterious sequence variants in the genome. Both variants were analyzed using in silico bioinformatic tools and were scored as 'uncertain significance' based on the current ACMG guidelines ${ }^{32}$ (Online Supplementary Figure S1). Both TUBB1 variants are positioned towards the $C$-terminal region of $\beta 1$-tubulin as indicated in Figure $1 C$ and D. This region is positioned away from the dimer interface with $\alpha$-tubulin, and the glutamate rich C-terminal tail, not present in the model, is an established site for PTM (Figure 1C and D). ${ }^{1}$ Both affected TUBB1 sequence variants are highly conserved in mammals (Figure 1E). R359 is found within a loop region towards the C-terminus of tubulin, and we predict mutating this residue would not cause dramatic misfolding within the protein secondary structure. R359 does form direct polar contacts with the $\mathrm{N}$-terminal helix and removing this contact through the sequence variant to the hydrophobic tryptophan may result in minor structural changes to the connected C-terminal regions, potentially affecting PTM or interactions with critical microtubule accessory proteins (MAP) (Figure $1 \mathrm{C}$ and D). Similarly, the $G$ insertion and subsequent frameshift effectively deletes the C-terminus of the protein and would therefore, if the protein folds correctly, result in effects similar, or more extreme, to the substitution of R359.

Patient $\mathrm{B}$ demonstrated a significant reduction in surface P-selectin expression and fibrinogen binding by flow cytometry in response to all agonists tested (Online Supplementary Figure S1). Family A showed no change in the levels of surface receptor expression, but weak Pselectin and fibrinogen responses when activated with a low concentration ADP, CRP, and PAR-1, suggesting a mild functional defect (Online Supplementary Figure S1C). Patient B also showed marked reduced expression of Pselectin and fibrinogen uptake in response to the same activation agonists (Online Supplementary Figure S1D and E). Patients with C-terminal variants in this study and others previously reported by Fiore et al. present with a macrothrombocytopenia also found in individuals with a complete loss of the $\beta 1$-tubulin, suggesting that the $\mathrm{C}$-ter- minal tail is likely critical to the function of $T U B B 1$ in the roles of microtubules in $\mathrm{MK}$ and platelets. ${ }^{18-20}$ As this C-terminal tail is rich in glutamate residues which are often targeted for polymodification, we began to investigate the polymodification of $\beta 1$-tubulin.

\section{C-terminal variants of $\beta 1$-tubulin fold correctly and demonstrate a reduction in polymodification}

First, we investigated the effects of the two patient variants (p.R359W and p.L361Afs*19) on the folding and potential polymodification of $\beta 1$-tubulin. We designed, generated, and validated a $\beta 1$-tubulin-mApple fusion construct (Online Supplementary Figure S2). This plasmid was further mutated to harbor each of the patient variants (p.R359W and p.L361Afs*19), and an artificial C-terminal truncation which specifically deletes the glutamate rich Cterminal tail (Figure 2A; Online Supplementary Figure S2). The WT $\beta 1$-tubulin construct was transfected into Hek293T cells and co-stained for polyglutamylated and polyglycylated tubulin specifically. Transfected cells are exclusively positive for both residues indicating that $\beta 1$ tubulin is indeed polymodified (Figure 2B). Expression of each of the mutated constructs show that both patient variants and the C-terminal truncation fold correctly (Figure 2C). Furthermore each of them results in a consistent and significant reduction in polymodification (Figure 2D). This data indicates that both patient variants result in a correctly folded $\beta 1$-tubulin which has a similar effect to a truncation of the C-terminus, and is further supported by western blotting of mutant constructs (Online Supplementary Figure S3) which show a significant reduction in polymodification.

\section{Induced pluripotent stem cell-derived proplatelet forming megakaryocytes are both polyglycylated and polyglutamylated}

The tubulin code posits that a highly lineage and species specific expression of modifying enzymes mediates cell specific PTM. While our transfection data successfully indicates that $\beta 1$-tubulin is indeed polymodified, and that each of our patient genetic variants result in the expression of a functional $\beta 1$-tubulin with a C-terminal truncation, data from both human MK and platelets is needed to dissect the role of polymodification in these cells.

To date, polyglycylation has not been reported in either platelets or MK. Polyglutamylation has recently been reported in a modified $\mathrm{CHO}$ cell line and human platelets. In order to investigate polymodification in human $\mathrm{MK}$, we adapted a directed differentiation protocol previously reported by Feng et al. to generate large populations of mature, proplatelet forming cells (Online Supplementary Figure S4). iPSC-MK were stained for CD42b as a marker for mature, and hence TUBB1 expressing, MK, and both polyglutamylated and polyglycylated tubulin. CD42b+ cells were found to be positive for both polyglutamylated tubulin and polyglycylated tubulin (Figure 3A), while neighboring cells in the sample negative for CD42b did not demonstrate these polymodifications (Figure 3B). Across multiple differentiations we consistently yielded a purity of approximately 50-60\% CD42b+ cells (Figure 3C), which on analysis are positive for both polyglutamylated and polyglycylated tubulin (Figure 3D). Finally, $100 \%$ of proplatelet forming cells observed across replicates were positive for both polymodifications (Figure $3 \mathrm{E}$ ), and this was further confirmed by western blotting (Figure 3F). 
A

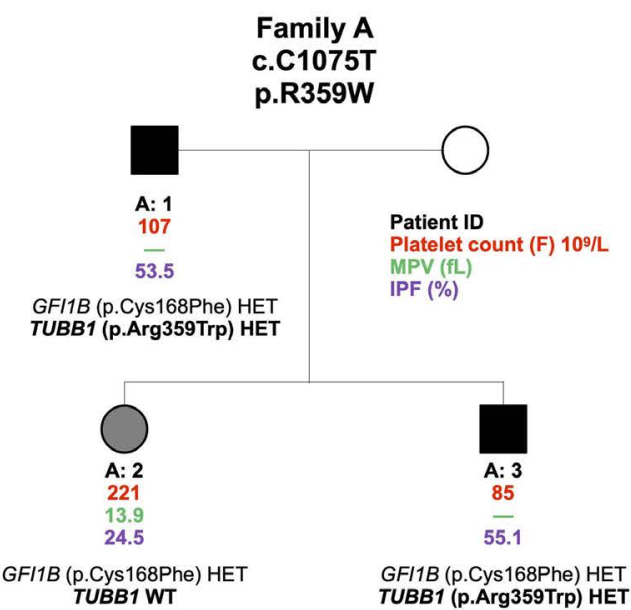

Patient B

c.1080 1081insG p.L361Afs*19

\section{Control}

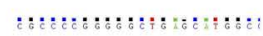

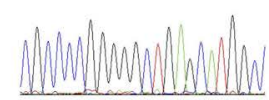

\section{p.L361Afs*19}
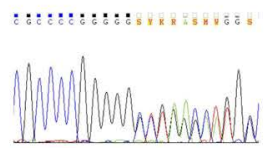

B

\begin{tabular}{|l|l|l|l|l|l|l|l|l|}
\hline Patient & Age & Sex & TUBB1 Mutation & GFI1B Mutation & Plat. count (x109/L) MPV (fL) & IPF (\%) & Secondary defect \\
\hline A: 1 & 66 & M & p.R359W & p.C168F & 107 & large & 53.5 & Yes \\
\hline A: $\mathbf{2}$ & 41 & F & WT & p.C168F & 221 & 13.9 & 24.5 & Yes \\
\hline A: $\mathbf{3}$ & 29 & M & p.R359W & p.C168F & 85 & large & 55.1 & Yes \\
\hline B:1 & Dec. & M & p.L361Afs 19 & None & 11 & $13.4+$ & N/A & Yes \\
\hline
\end{tabular}

TubB1 C-Terminal Tail

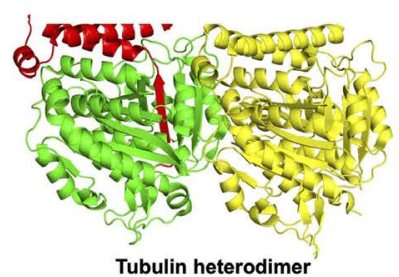

D

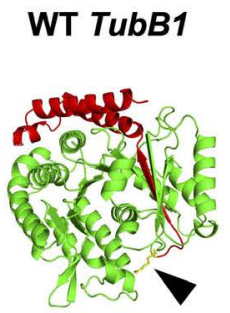

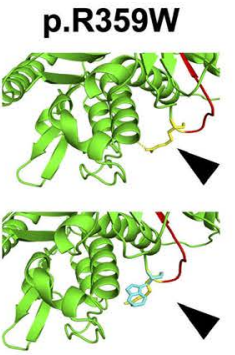

p.L361Afs*19

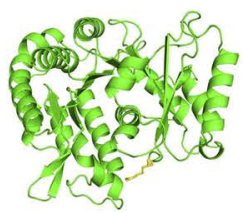

TubB1 C-Terminal Tail:

VLEEDEEVTEEAEMEPEDKG

E

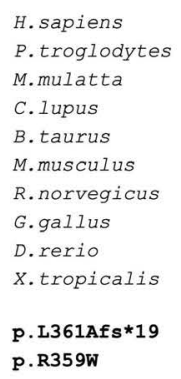

H. sapiens

P.troglodytes

M. mulatta

C. Iupus

B.taurus

M. musculus

R. norvegicus

G.gallus

D.rerio

X.tropicalis

p. L361Afs $* 19$

p. R359W

NVKVAVCDIPPRGLSMAATF I GNNTA IQE I FNRVSEHF SAMFKRKAFVHW... NVKVAVCDI PPRGLSMAATF I GNNTA IQE I FNRVSEHF SAMFKRKAFVHW... NVKVAVCDIPPRGLSMAATFIGNNTA IQE IFNRVSEHFSAMFKRKAFVHW.. NVKVAVCDIPPPGLSMAATFMGNSTAVQELFSRVAEHFSAMFRRRAFVHW. NVKVAVCDIPPRGLSMAATF IGNNTA I QELFSRISEHF SAMFKRKAFVHW... NVKVAVCDIPPRGLNMAATF LGNNTAIQE LFTRVSEHFSAMFRRRAFVHW... NVKVAVCDIPPRGLNMAATFLGNNTAIQELFTRVSEHFSAMFRRRAFVHW... NVKVAVCDIPPRGLKMAATF IGNNTA IQE LF IRVSEQFSAMFRRKAFLHW... NVKVAVCDIPPRGLKMASTFIGNNTAIQEVFKRIGEQFALMFRRKAFLHW... NVKVAVCDIPPRGLKLASTFIGNNTAIQE IFRRIADQFSAMYRRKAFLHW..

NVKVAVCDIPPRGAEHGRHLHWQGHGHPRDI* NVKVAVCDI PPWGLSMAATFIGNNTAIQEIFNRVSEHFSAMFKRKAFVHW..

Figure 1. Candidate TUBB1 variants and their hypothesised effect on the C-terminus of $\beta 1$-tubulin. (A and B) Two unrelated families were identified as carrying genetic variations in the TUBB1 gene within six base pairs of one another. The first, family A, is comprised of three individuals, two of whom carry an arginine to tryptophan (p.R359W) coding variant. Interestingly, all three individuals in family A harbor an additional GFI1B variant. However, the individuals with the reported TUBB1 variant (A:1 and $A: 3$ ) present with a macrothrombocytopenia and high immature platelet fractions (IPF), while the patient without the R359W TUBB1 variant presented with a normal platelet count. The second family is comprised of a single individual, recently deceased, with a frameshift variant six base pairs from the missense change reported in family A. In this individual's case, the insertion of a guanine nucleotide results in a frameshift with a premature stop codon 19 amino acids from the leucine to alanine change. Platelet count normal range 147-327x10\%, $(n=40)$, mean platelet volumes (MPV) normal range 7.8-12.69 fL ( $n=40)$, immature platelet fraction (IPF) normal range 1.3-10.8\% $(n=40)$. (C) The C-terminal tail is downstream of both genetic variants in these families, and projects away from the dimer:dimer interface. The C-terminal sequence of TUBB1 is rich in glutamate residues which can be targeted for polymodification. (D) Based on homology modeling of TUBB1, we predict that the missense variant reported in family $\mathrm{A}$ is likely to affect the fold of the C-terminal tail, while the frameshift causes a truncation of this region. (Black arrows indicate the position of the $\mathrm{R} 359$ residue). (E) The arginine residue substitution in family $A$ is highly conserved across species, as are sequences adjacent to the frameshift in patient $B$. 
CRISPR knockout of TUBB1 results in a complete loss of proplatelet formation

To date, the loss of TUBB1 has not been studied in human MK. We generated an iPSC line with a CRISPR mediated bi-allelic loss of function mutation in the N-terminus of the coding region of TUBB1 (Figure 3G; Online Supplementary Figure S5). The mutation of the TUBB1 start codon on both alleles results in a complete loss of expres- sion (absence of T2 band in Figure $3 \mathrm{H}$ ) and proplatelet formation in vitro (Figure 3 I and J, right panel). Unfortunately, our attempts to generate C-terminal truncations through CRISPR in iPSC-MK were unsuccessful as multiple guides targeting the 3 ' end of the $T U B B 1$ gene failed to cleave the genomic sequence. Interestingly while TUBB1 KO clones stain positively for polyglutamylated and polyglycylated tubulin, the distribution of these residues is disturbed

A

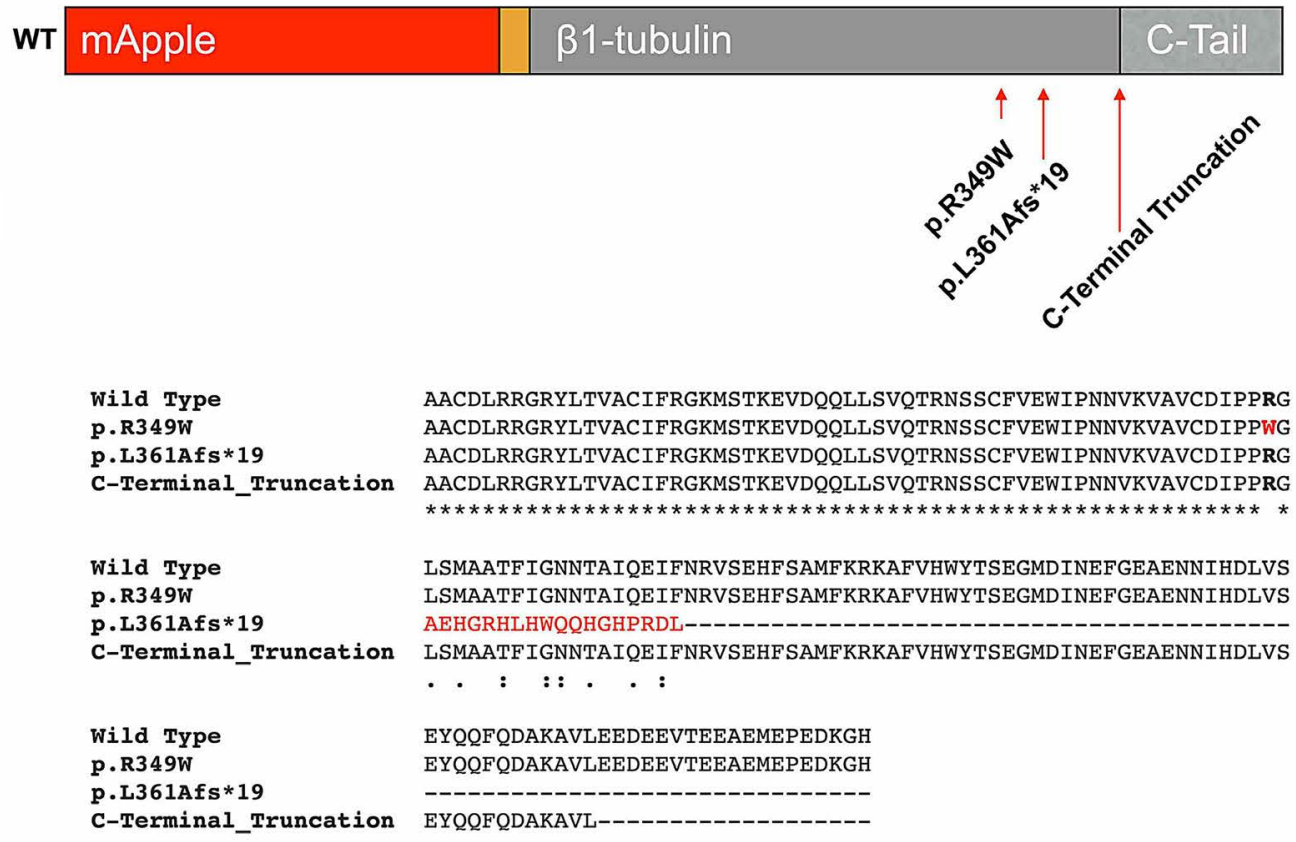

B
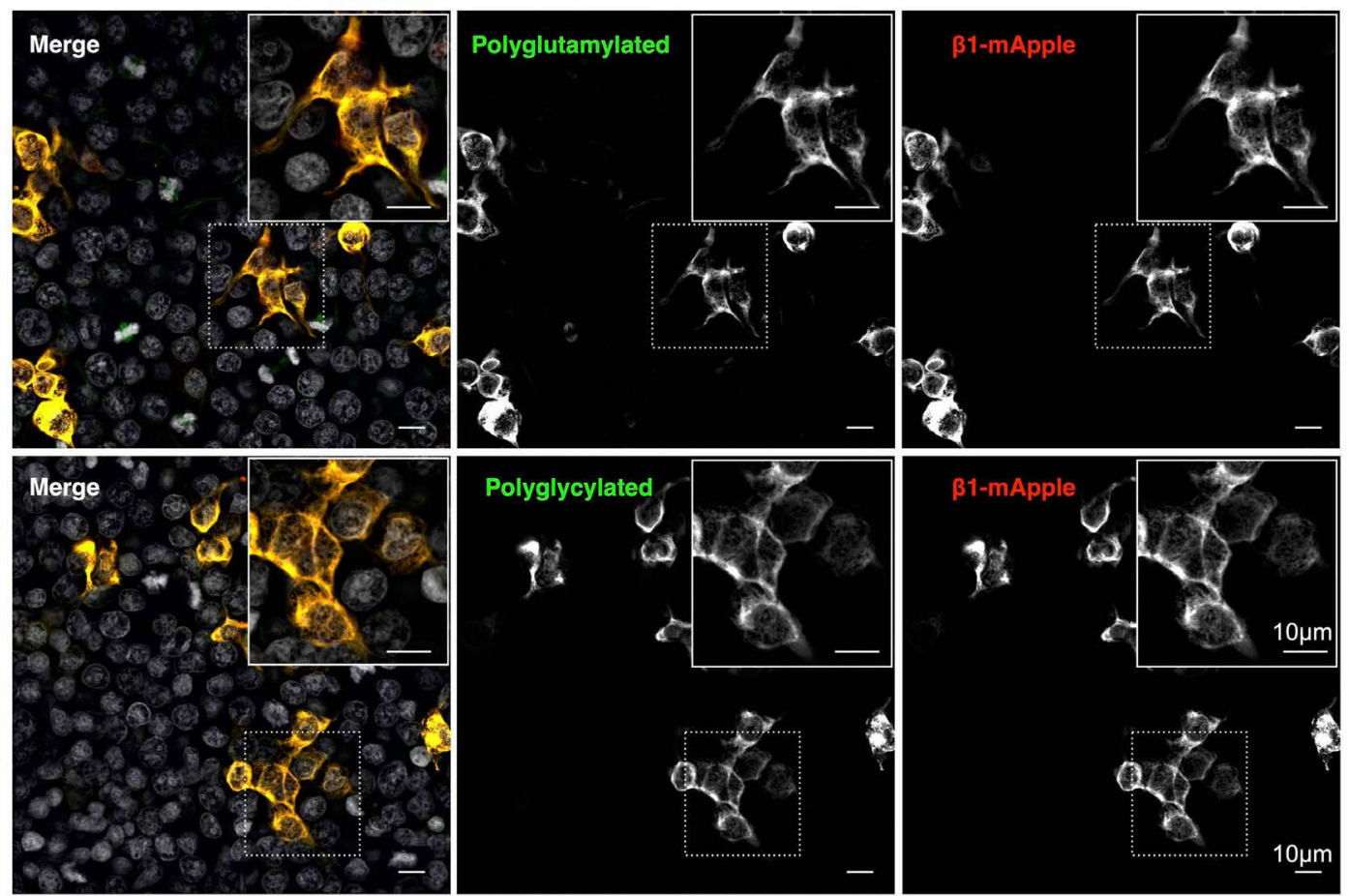

Figure 2. Continued on following page. 
C
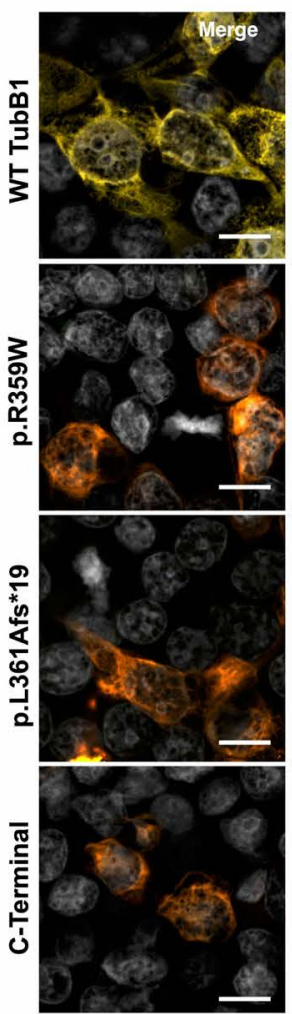
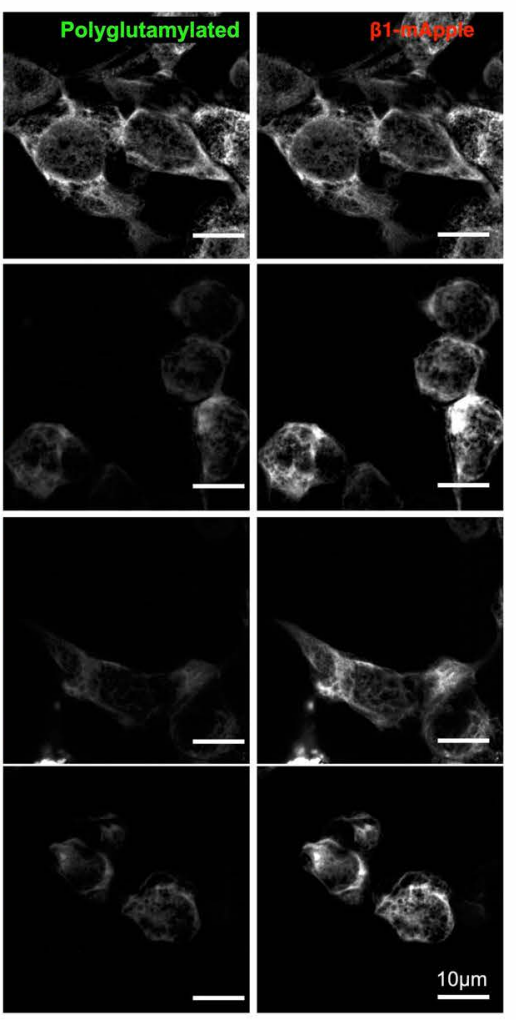

D
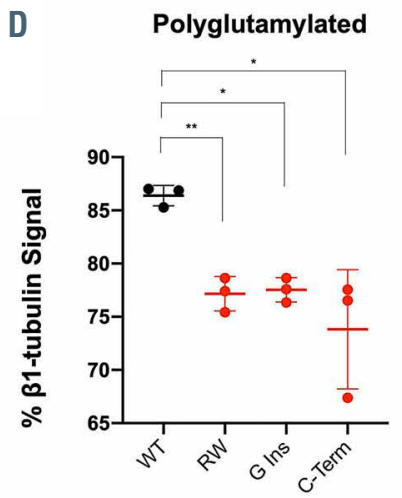

Polyglutamylated

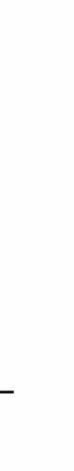

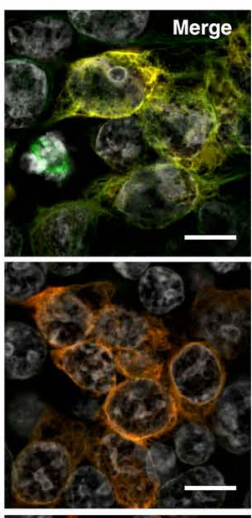
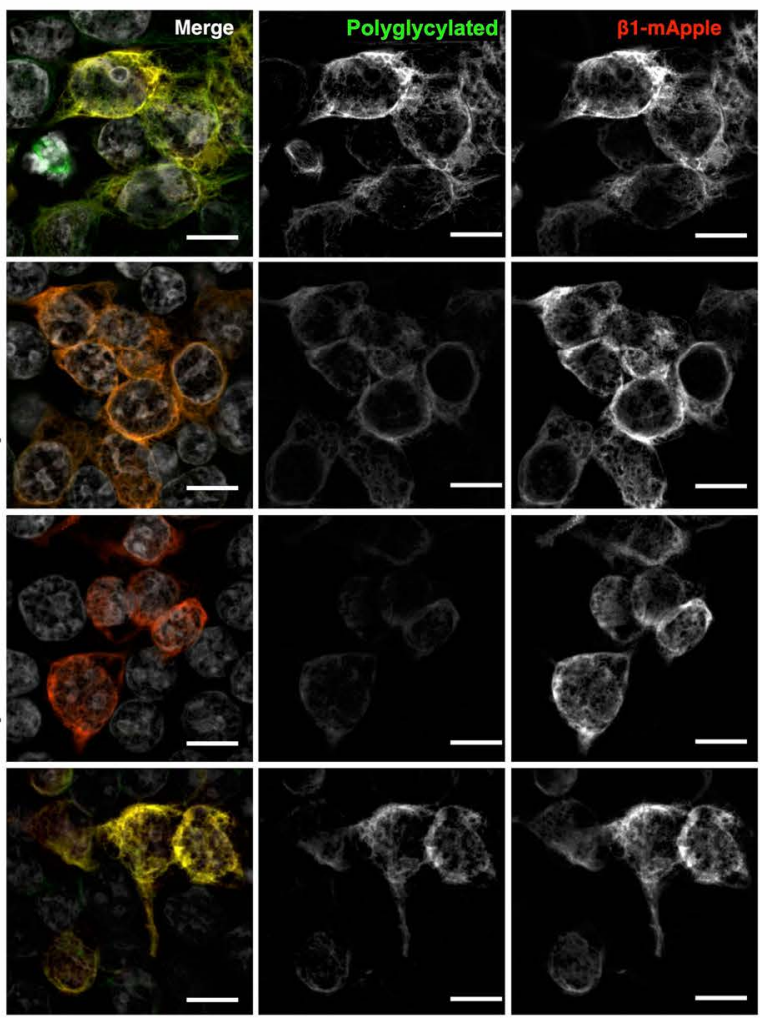

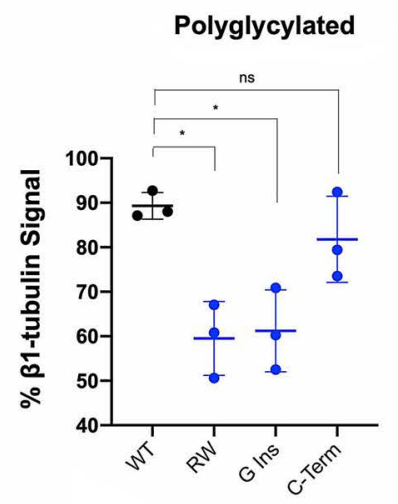

Figure 2. Cells expressing wild-type and mutated $\beta 1$-tubulin demonstrate C-terminal polymodification. (A) Constructs carrying wild-type (WT), patient variants (p.R359W and p.L361Afs*19), and a C-terminal tail truncation of $\beta 1$-tubulin fused to the fluorescent reporter mApple at the N-terminus were designed and cloned. (B) The WT construct was first transfected into Hek293T cells, which were then fixed and immunostained for polyglutamylated and polyglycylated tubulin residues specifically. (C and D) A comparison of the WT and mutated constructs shows a reduction in polyglutamylation and polyglycylation in each mutant compared to the WT. ( $n=3$ independent differentiations, standard deviation plotted on graphs. Two-way ANOVA with multiple comparisons performed to establish significance.)

when compared to WT platelet forming iPSC-MK (Figure 3K). While polyglycylated and polyglutamylated residues form a distinct peripheral band around WT MK as shown in Figure 3K, the $\mathrm{KO}$ cells demonstrate a diffuse tubulin staining (evidenced by line profiles in Online Supplementary Figure S7). Figure 3L shows quantification of this staining whereby WT iPSC-MK display higher mean intensity than $\mathrm{KO}$ constructs in polyglycylated tubulin, however no significant quantitative change in polyglutamylation was observed. This is the first data demonstrating the effects of the loss of TUBB1 expression in human MK.

\section{Platelets demonstrate a different pattern of polymodification}

We hypothesized that the $\beta 1$-tubulin polymodifications evident in $M K$ might be differently regulated between resting and activated platelets. We therefore compared immunofluorescence staining of polyglutamylated and polyglycylated tubulin between resting platelets and cells spread on fibrinogen and collagen.

Resting platelets demonstrate polyglutamylated tubulin which partially co-localizes with the $\beta 1$-tubulin marginal band unlike MK which demonstrate extensive polyglycylation in proplatelet forming cells (Figure 4A). On fibrinogen and collagen spreading, polyglutamylation is evident, notably at the marginal band of spreading cells on fibrinogen (Figure 4B). A lack of polyglycylation is consistent in both resting and activated platelets. Western blotting of resting platelets and cells activated through stimulation by CRP over time does not show an increase in the total 
A
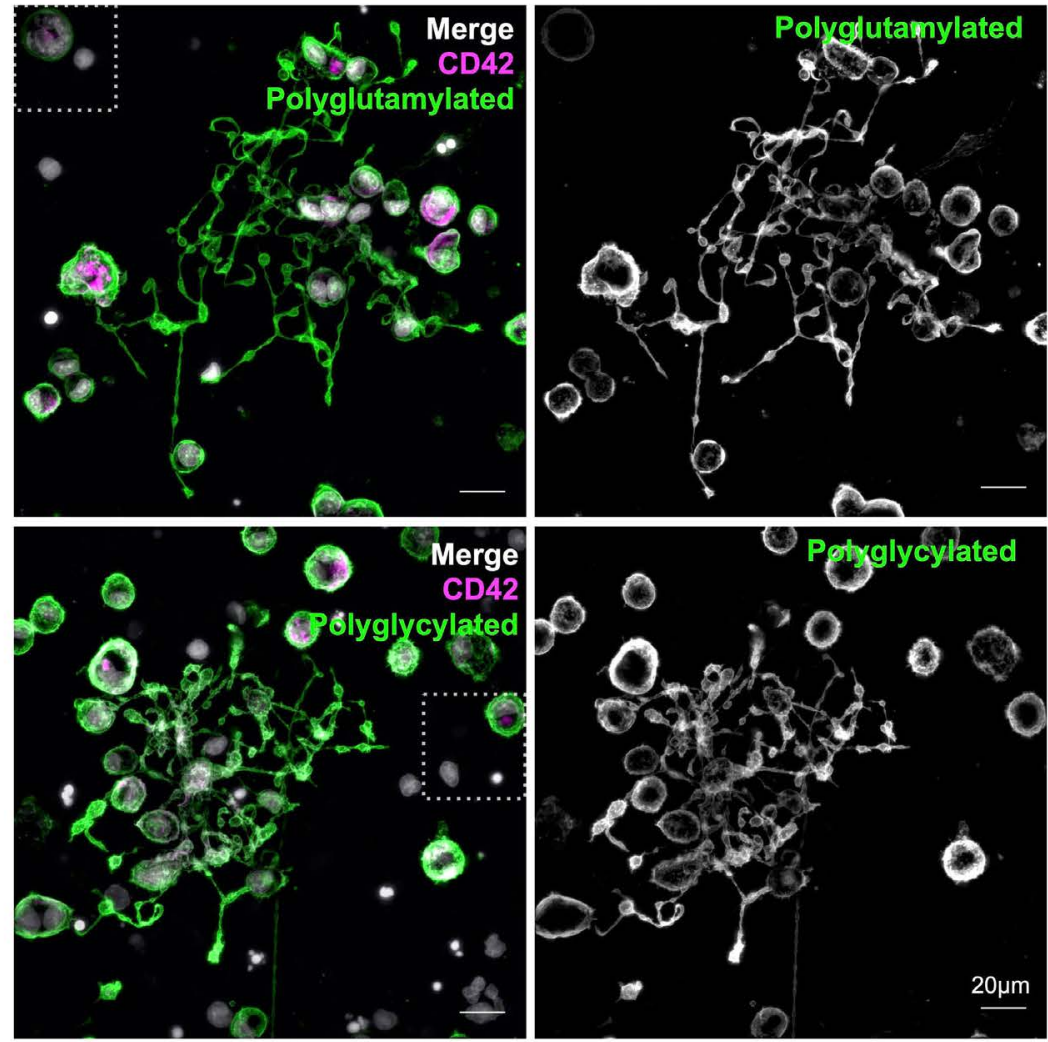

B
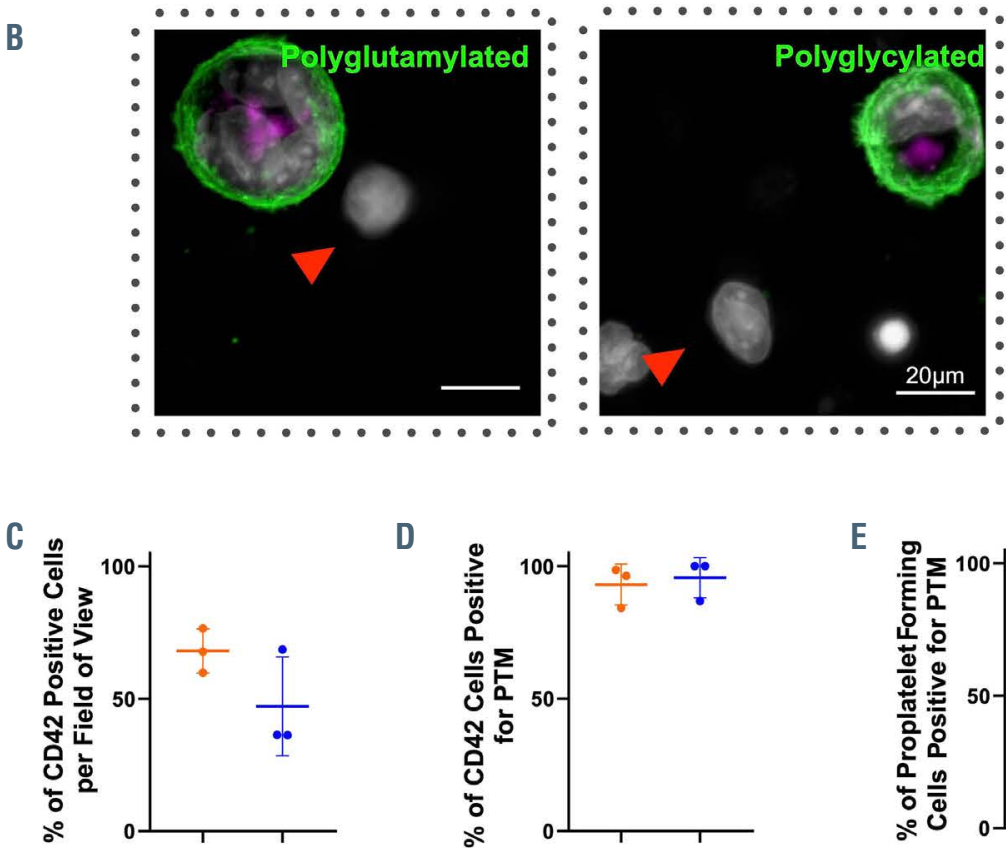

D

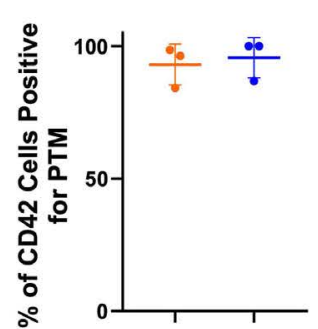

E

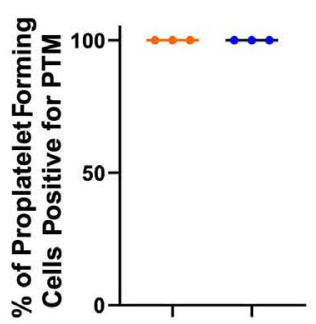

- Polyglutamylated tubulin - Polyglycylated tubulin

F

IPSC-MK

iPSC-MK
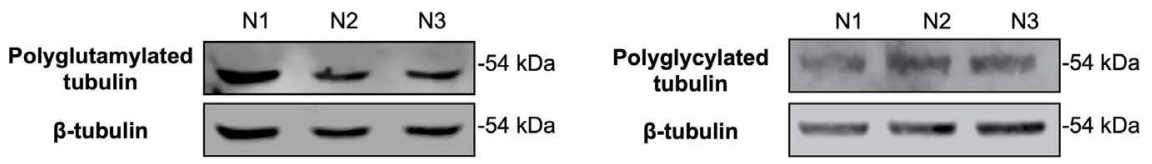

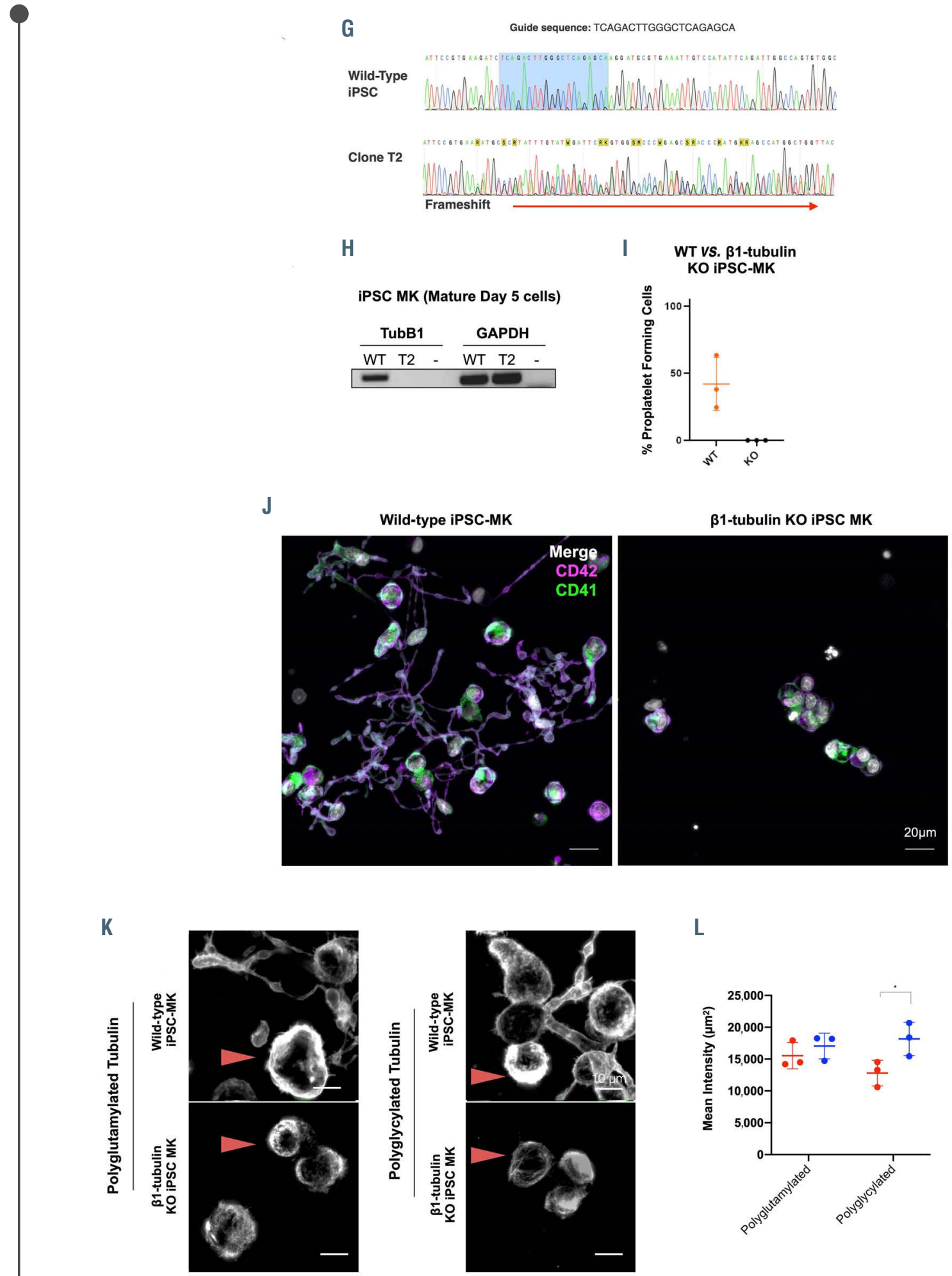

$\mathbf{L}$

Figure 3. Mature, proplatelet forming induced pluripotent stem cell megacarycytes are both polyglutamylated and polyglycylated. TUBB1 knockout (KO) iPSC-MK do not form proplatelets and demonstrate disordered polymodified tubulin. (A) iPSC-MK co-stained for CD42 and polyglycylated or polyglutamylated tubulin show that these cells are positive for both polymodifications. Both polyglutamylated and polyglycylated tubulin are evident in proplatelet extensions, including nascent platelet swellings on the proplatelet shaft. (B) Neighboring CD42b-cells are negative for both polymodifications (indicated by red arrows). (C and D) Approximately 50-60\% of cells in multiple differentiations are CD42b+, and these cells are 100\% double positive for polymodification and CD42b+. (E) All proplatelet extensions observed are positive for polymodification. (F) Polyglutamylation and polyglycylation are evident by western blotting in mature iPSC-MK derived from three separate differentiations. (G) iPSC were transfected with a TUBB1 targeting guide RNA, after which indel positive cells were isolated and sequenced to positively identify a bi-allelic insertion-deletion mutant (clone T2). (H) This clone was further analyzed and loss of $\beta 1$-tubulin expression was confirmed by quantitative real-time polymerase chain reaction (qRT-PCR). (I and J) A comparison of proplatelet production in wild-type (WT) vs. TUBB1 KO cells revealed a complete loss of proplatelet formation in mutant CD41/42b+ cells. (K) TUBB1 KO clones show a disordered arrangement of polymodified residues when compared to WT cells. KO cells do not demonstrate the reorganization of tubulin to the periphery of the cell evident in WT IPSC-MK (indicated by red arrows). (L) A measure of the polyglutamylation and polyglycylation in WT vs. KO clones reveals a significant increase in polyglycylation in mutant cells consistent with an aberrant accumulation of these residues. ( $n=3$ independent differentiations, standard deviation plotted on graphs). 
amount of polyglutamylated tubulin (Figure 4C). An increase in the co-localization between $\beta 1$-tubulin and polyglutamylated residues suggests that while the total polyglutamylation of platelets remains unchanged through activation, the distribution of these residues is altered (Figure 4D). We confirmed the polyglutamylation (and loss of polyglycylation) in microthrombi generated by CRP stimulation (Figure $4 \mathrm{E}$ to $\mathrm{G}$ ). This data shows that while polyglutamylation and polyglycylation are evident in platelet producing iPSC-MK, polyglycylation is completely lost in mature platelets. This is the first evidence of a system whereby these competitive polymodifications are dramatically altered between the 'parent' and terminal cell, suggesting markedly different roles for these residues in mediating the function and activity of $\beta 1$-tubulin. Acetylation and tyrosination have been previously reported in platelets, however, their role in maintaining the marginal band and/or driving morphological change on platelet activation remains unclear. ${ }^{13}$ In order to determine whether the polyglutamylation of the marginal band we observe thus far coincides with these PTM, we performed a time course of spreading on fibrinogen to determine whether there is an equivalent increase in either acetylation or tyrosination of the marginal band. Interestingly, we found a significant decrease in acetylation and tyrosination over time (between 0 and 10 minutes), while a notable polyglutamylation of the marginal band is evident from the earliest time point (10 minutes spreading on fibrinogen) and does not decrease (Figure $4 \mathrm{H}$ and I).

\section{Platelet and megakaryocyte polymodifications regulate} motor protein localization to drive both proplatelet formation and platelet shape change on activation

Polyglutamylation has been reported as a means by which motor protein processivity is regulated, and like in neuronal cells, MK proplatelet formation is known to be
A
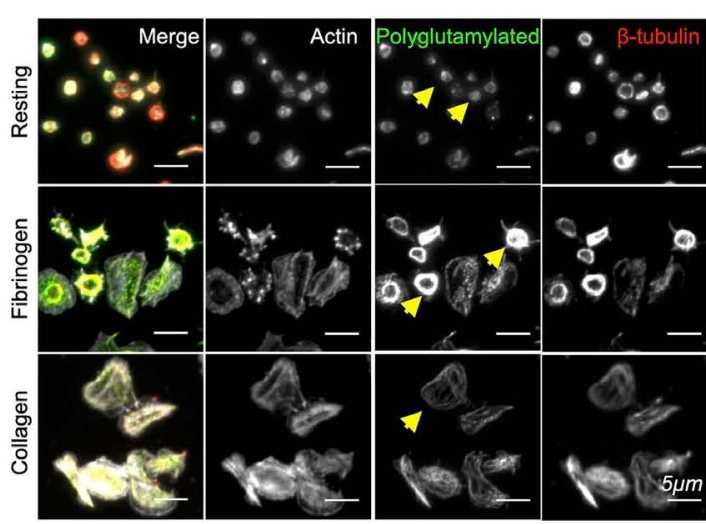

C

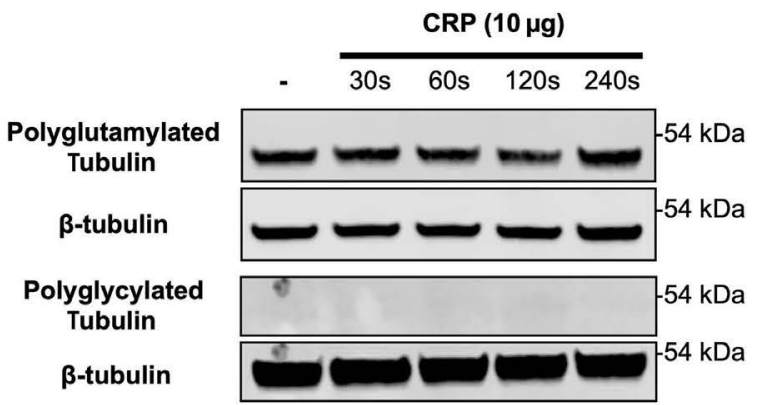

E

Mean intensity of post-translationally modified tubulin residues on spreading

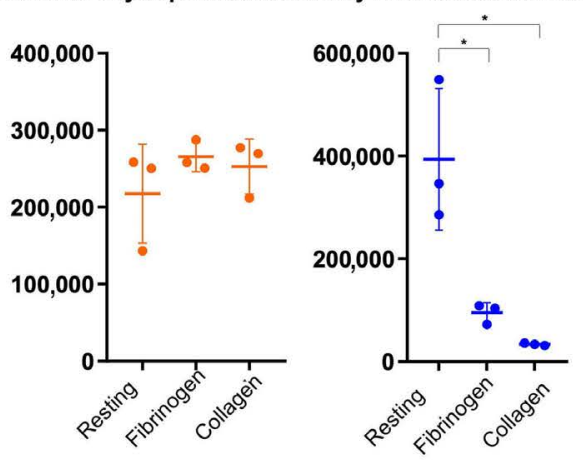

B

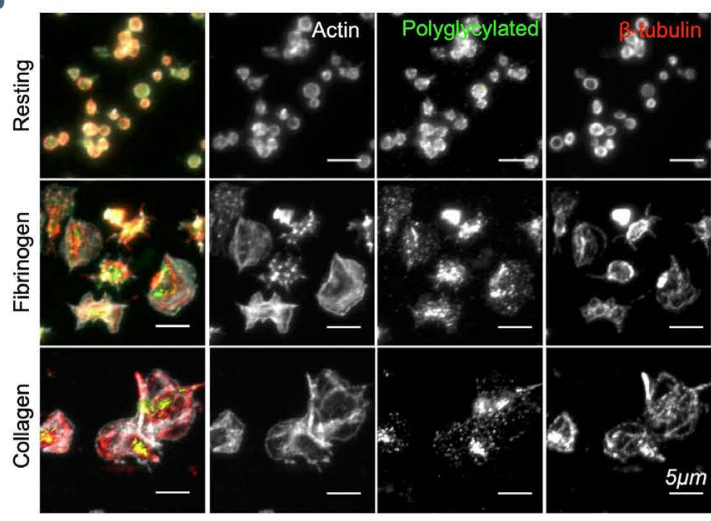

D

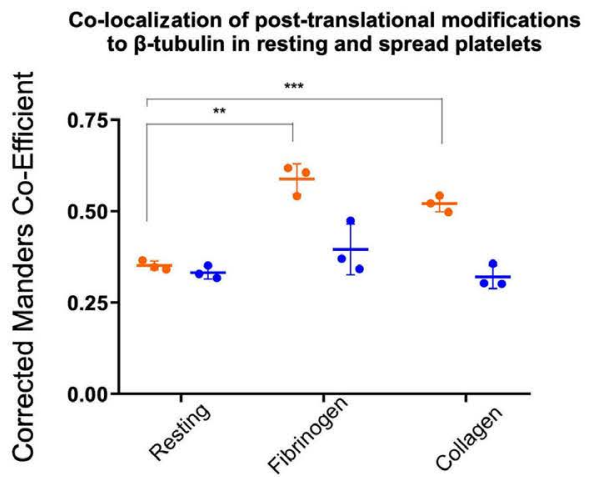

- Polyglutamylated Tubulin

- Polyglycylated Tubulin

Figure 4 Continued on following page. 
F

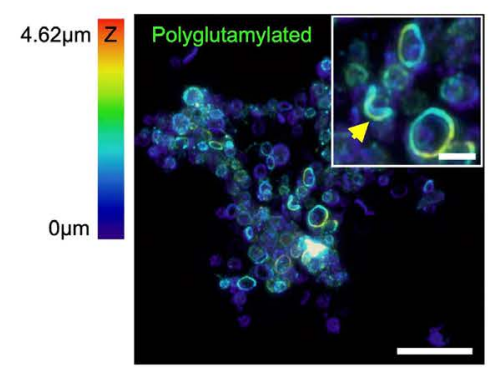

G
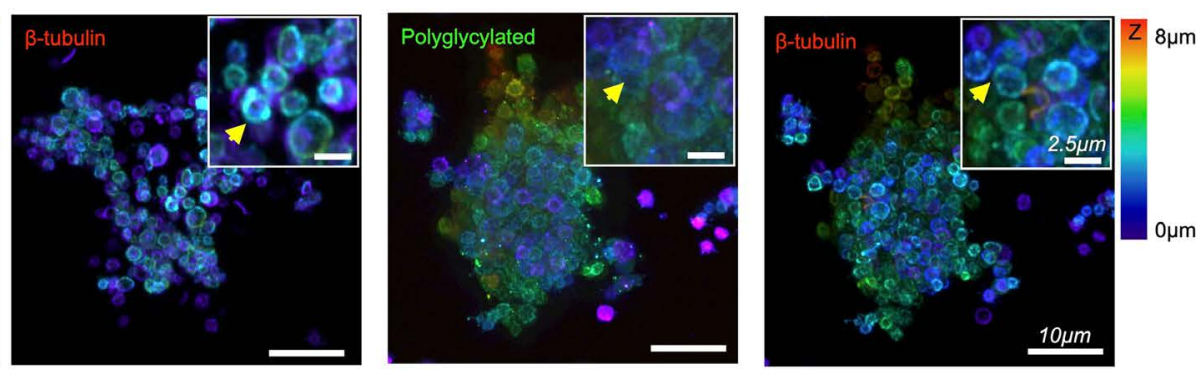

H

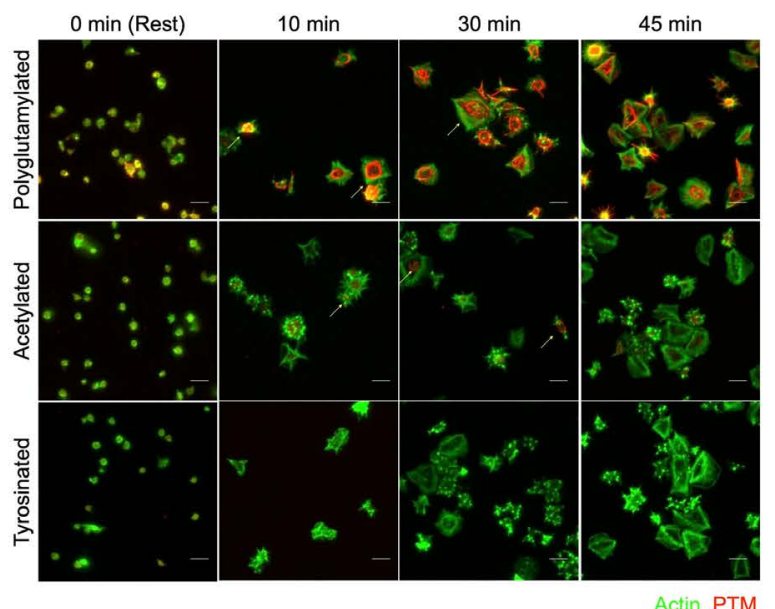

$\rightarrow$ Polyglutamylated Tubulin

- Acetylated Tubulin

- Tyrosinated
| Change in mean intensity over time

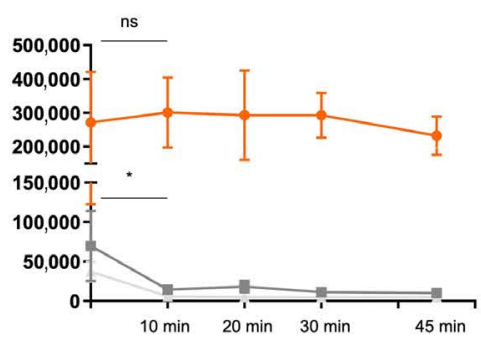

Figure 4. Platelet activation results in polyglutamylation of the marginal band. (A) Resting platelets show a partial polyglutamylation of the marginal band and a loss of the polyglycylation evident in induced pluripotent stem cell megacaryocytes (iPSC-MK). (B) Platelet spreading on fibrinogen and collagen shows an accumulation of polyglutamylation at the marginal band as platelets spread, and no evidence of polyglycylation. (C) Western blotting of resting and CRP activated platelets confirms the presence of polyglutamylated tubulin and a loss of polyglyclation. No increase in polyglutamylation is evident over time. (D, E) A measurement of colocalization (corrected Manders coefficient) between polyglutamylated tubulin and $\beta 1$-tubulin in resting and spread platelets shows a significant increase in the colocalization of these modified tubulin residues on platelet activation and spreading. (F, G) Platelets activated in vitro using CRP were fixed and co-stained for $\beta 1$-tubulin and polyglutamylated residues, and then imaged in $3 \mathrm{D}$ using AiryScan confocal (stacks colorized in Z as indicated by the color chart in this figure). In these microthrombi, polyglutamylation of the marginal band is evident, while polyglycylation is not observed. $(\mathrm{H}) \mathrm{A}$ time course was performed to compare polyglutamylated tubulin with two other previously reported post-translational modifications (PTM) in platelets (acetylation and tyrosination). Polyglutamylation is maintained over time, while acetylation and tyrosination decrease significantly as platelets spread. (I) The mean fluorescence intensity of polyglutamylated tubulin is markedly higher than either acetylated or tyrosinated tubulin. ( $n=3$, standard deviation. Two-Way ANOVA with multiple comparisons. $10 \mu \mathrm{m}$ scale bar.)

driven by a mechanism of dynein mediated proplatelet sliding. ${ }^{33}$ Similarly, the antagonistic movement of dynein and kinesin are known to maintain the marginal band in resting platelets. ${ }^{13}$

In order to test if polymodification affects the spatial distribution of motors, we performed a time course of platelet spreading on fibrinogen and measured co-localization between polyglutamylated tubulin and dynein (DNAL1 - axonemal light chain 1). We observe a loss of co-localization between dynein and polyglutamylated residues upon platelet spreading (Figure $5 \mathrm{~A}$ and $\mathrm{B}$ ). Interestingly, axonemal dynein is also localized towards the leading edge of spread platelets (Figure 5A). This data suggests that the increased polyglutamylation of the marginal band observed on platelet spreading drives an outward movement of axonemal dynein. In order to investigate the role of axonemal dynein specifically in this process, we also stained spreading platelets for cytoplasmic dynein and observe a central distribution, suggesting an alternative role for cytoplasmic dynein in platelets (Online Supplementary Figure S6).

The loss of co-localization between polyglutamylated tubulin and dynein was confirmed on both collagen and fibrinogen spread platelets (Figure $5 \mathrm{C}$ and $\mathrm{E}$ ). This spatial relationship was also observed between polyglutamylated tubulin and kinesin-1, a motor protein recently reported to be important in platelet secretion on platelet spreading (Figure $5 \mathrm{D}$ and $\mathrm{F}$ ). ${ }^{34}$

This data suggests that polyglutamylated tubulin is involved in localizing motor proteins during platelet activation and spreading. The actions of kinesin and dynein are known to be critical to driving platelet shape change on activation, and this work is consistent with previous reports of polyglutamylated tubulin altering the processivity of motors in axons.

We then investigated the role of these polymodifications on the distribution of motors in iPSC-MK. In proplatelet extensions axonemal dynein and kinesin- 1 are both evident along the length of the proplatelet shaft (Figure 5G). $\beta 1$-tubulin $\mathrm{KO}$ cells show no proplatelet formation, and a significant reduction in the co-localization of dynein with polyglutamyulated residues when compared to WT iPSC-MK (Figure 5H and I). No significant change in the co-localization of these residues with kinesin-1 is observed between WT and KO iPSC-MK (Figure 5J). 
Megakaryocyte and platelet polymodification is regulated through the expression of both modifying and reversing enzymes

We hypothesized that the expression of cell specific subsets of effecting (tubulin tyrosine ligase like [TTLL]) and reversing (cytosolic carboxypeptidase [CCP]) enzymes are required to achieve the distinctive polymodification we observe in $\mathrm{MK}$ and platelets. We designed a quantitative real-time polymerase chain reaction (qRT-PCR) panel to interrogate the expression of the 13 known mammalian TTLL and six CCP. We generated RNA from iPSC-MK at different stages of maturation (Figures 6A; Online Supplementary Figure S4A). Day 1 (d1) cells are representative of a pool of hematopoeitic stem cells (HSC) and MK progenitors, while day 5 (d5) cells are comprised of $60 \%$ CD41/42b+ cells (Figures 6A; Online Supplementary Figure
S4E). Finally, d5 cells treated with heparin to induce proplatelet formation ( $\mathrm{d} 5+\mathrm{Hep})$ were used to interrogate whether there is any specific upregulation of TTLL and/or CCP on proplatelet formation (Figures 6A; Online Supplementary Figure S4D).

GAPDH housekeeping controls for each of the three samples (d1, d5, d5+Hep) show equivalent amplification of the housekeeping control, while results for TTLL family proteins show a number of enzymes expressed at different levels across the maturation of these cells (Figure $6 \mathrm{~B}$ and $\mathrm{C}$ ). Candidate TTLL observed in the initial endpoint PCR were taken forward for quantification across replicates generated from multiple differentiations (Figure $6 \mathrm{C}$ and D). We find a significantly increased expression of TTLL1, TTLL2, TTLL4, and TTLL10 on proplatelet formation in cells treated with heparin $\left({ }^{* *} P=0.0081,{ }^{*} P=0.0105,{ }^{*} P=0.0260,{ }^{* *} P=\right.$
A

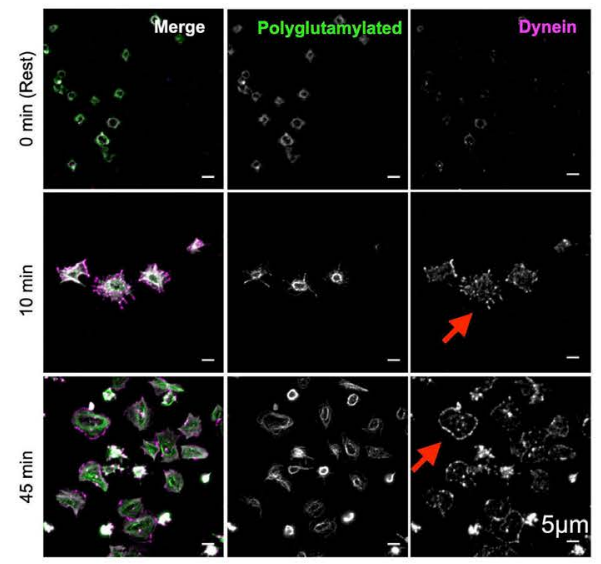

B Change in dynein co-localisation with

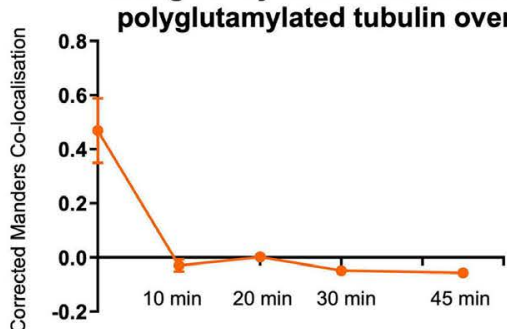

C

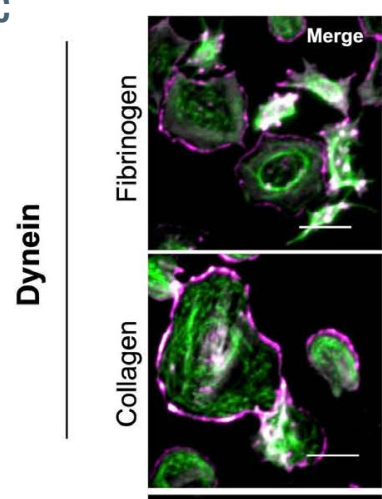

D

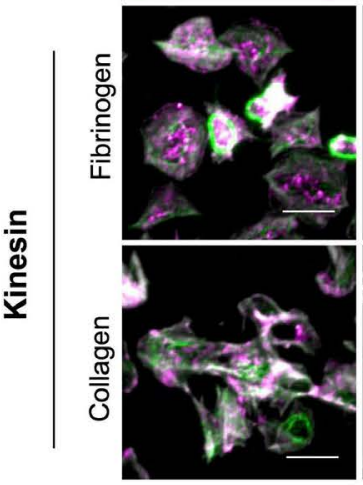

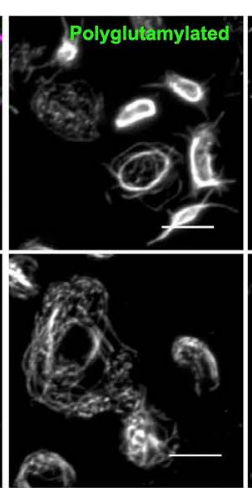
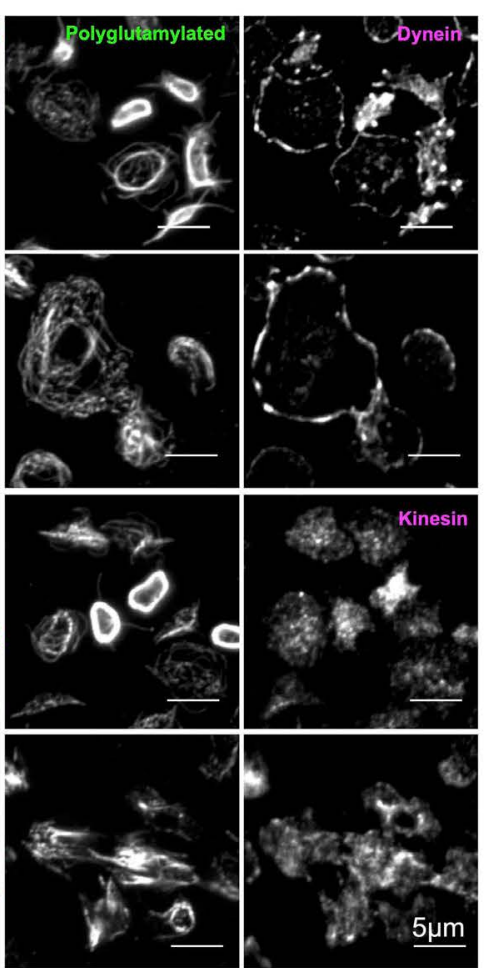

E

Co-localization of C-terminal PTM to Dynein in resting and spread platelets

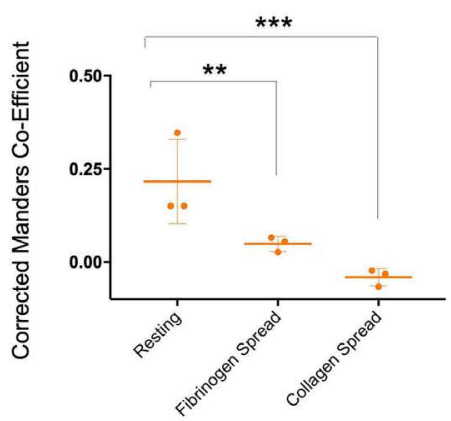

$\mathbf{F}$

Co-localization of C-terminal PTM to Kinesin in resting and spread platelets

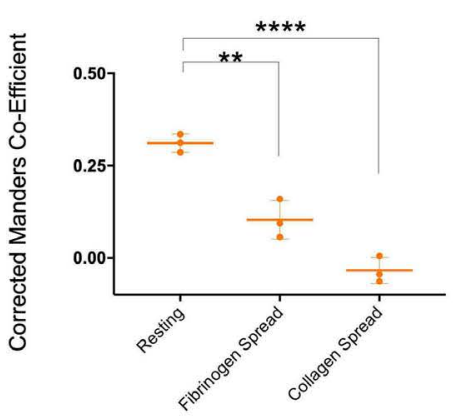


G

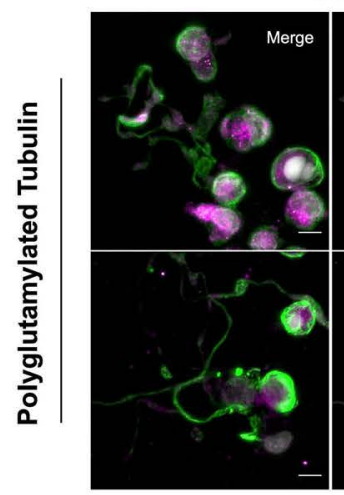

Wild-type iPSC MK
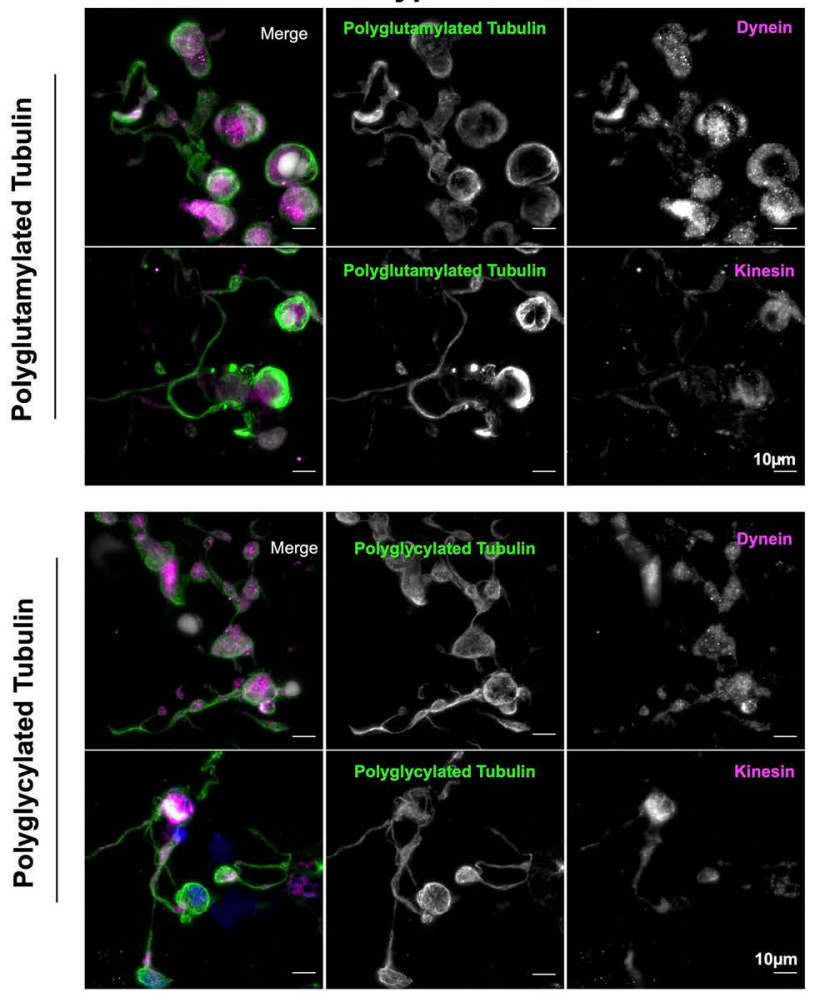

I

Co-localisation of C-terminal PTM to Dynein in WT and $\beta 1$ Knockout iPSC MK

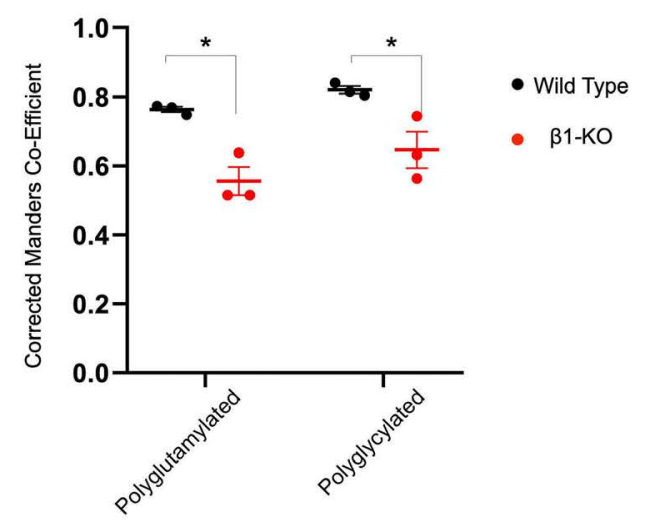

H
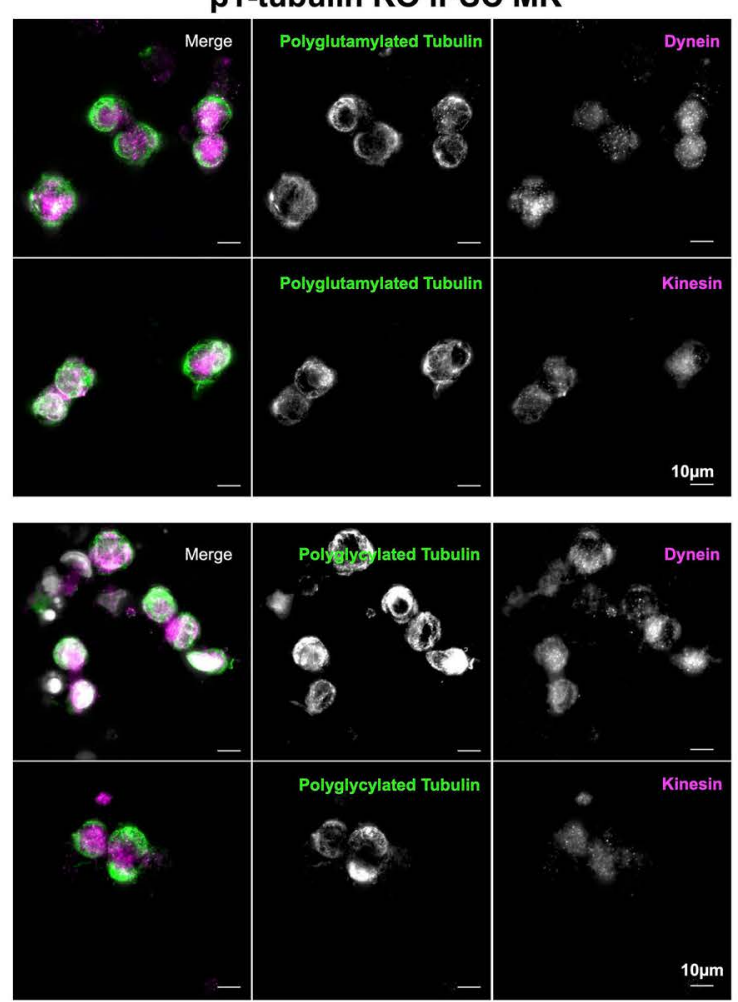

J Co-localisation of C-terminal PTM to Kinesin in WT and $\beta 1$ Knockout iPSC MK

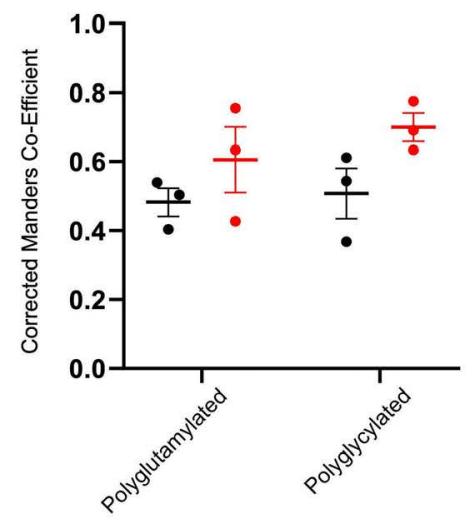

Figure 5. Polyglutamylation regulates spatial distribution of motor proteins in platelets and megakarycytes. (A and B) Quantification of the co-localization of dynein, as measured by the corrected Manders coefficient, with polyglutamylated tubulin over time shows a marked decrease in co-localization between these polymodified residues and the motor over time. (C) In fibrinogen and collagen spread cells, dynein is observed on the periphery of the spread cells, (D) while kinesin-1 is evident as a diffuse punctate stain. (E) Dynein co-localization with polyglutamylated residues decreases dramatically on platelet spreading in both fibrinogen and collagen $(* * P=0.0082$ and $* * * P=0.0004$ respectively). $(F)$ A loss of co-localization with polyglutamylated tubulin is evident in fibrinogen and collagen spread cells ( $* * P=0.0017, * * * * P<0.0001$ respectively). (G) Immunofluorescence staining of induced pluripotent stem cell megacaryocytes (iPSC-MK) for polymodified tubulin, dynein, and kinesin- 1 show the distribution of both motors along the length of the proplatelet shaft in wild-type (WT) cells. (H and I) In $\beta 1$-tubulin knockout (KO) iPSC$\mathrm{MK}$, no proplatelet extensions are formed and a significant reduction in the co-localization of dynein to polyglutamlated residues is observed $(* P=0.0166$ and $\star P=0.0293$ respectively), $(\mathrm{J})$ with no significant change in the co-localization of kinesin-1 with polyglycylated tubulin. ( $\mathrm{n}=3$, standard deviation. Two-Way ANOVA with multiple comparisons.)

0.0004 respectively) (Figure 6D). CCP family enzymes were also found to be expressed in maturing MK, notably CCP1, $3,4,5$, and 6 (Figure 6E), with CCP4 and CCP6 upregulated on proplatelet production $\left({ }^{*} P=0.0130,{ }^{* * *} P=0.0009\right)$ (Figure $6 \mathrm{~F}$ to $\mathrm{H})$.

In order to investigate TTLL and CCP expression in platelets, we repeated this panel on RNA extracted from resting and CRP stimulated donor platelets. We found that none of the TTLL and CCP observed in iPSC MK were consistently expressed across donors with the exception of TTLL7, a known polyglutamylase (Figure 6I, see complete gel in the Online Supplementary Figure S10)..$^{35}$ No differences between resting and activated platelets were observed (Figure 6I). This data shows a markedly different pattern of TTLL and CCP expression in both MK and platelets, correlating with the observed differences in polymodification. 
A

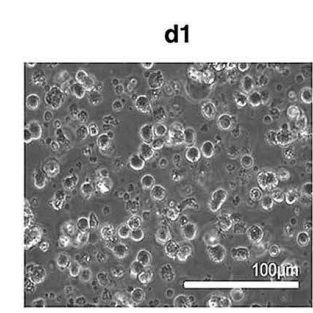

d5

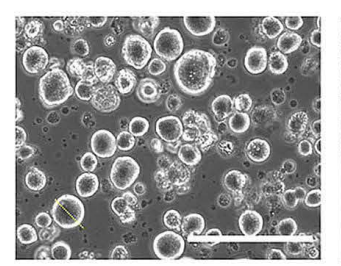

d5+Hep

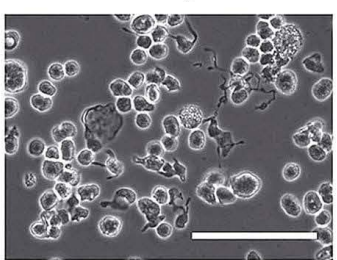

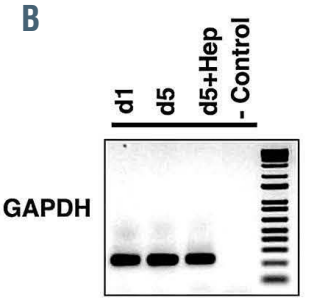

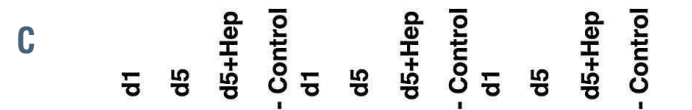

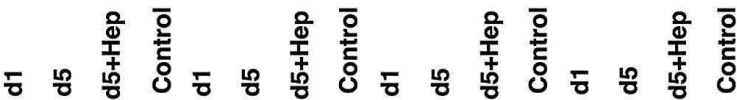

TTLL

\begin{tabular}{|c||c||c||}
\hline TTLL1(143bp) & TTLL2(138bp) & TTLL3(95bp) \\
\hline$-\infty$ & -- & -- \\
\hline
\end{tabular}

\begin{tabular}{|c||c||c||c|}
\hline TLL4 (106bp) & TTLL5(85bp) & TTLL6 (87bp) & TTLL7 (152bp) \\
\hline-- & $-\infty$ & $-\infty$ & $-\infty$ \\
\hline
\end{tabular}

\begin{tabular}{|c|c|c|c|c|c|}
\hline 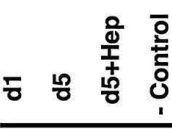 & 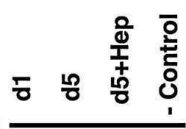 & 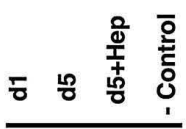 & 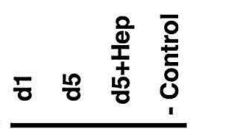 & 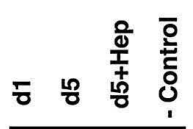 & 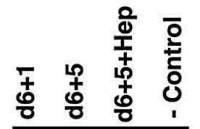 \\
\hline TTLL8 (174bp) & TTLL9 (158bp) & TTLL10 (90bp) & | TTLL11 (76bp) & TTLL12 (84bp) & TTLL13 (477BP) \\
\hline & & -- & ㅋ. & 40 & 0 \\
\hline
\end{tabular}

D TTLL family change in expression

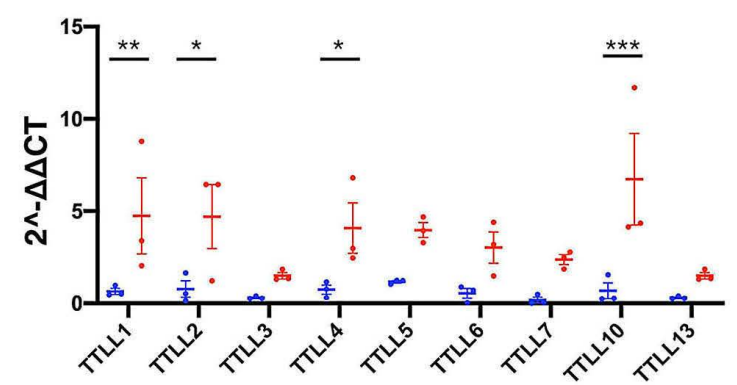

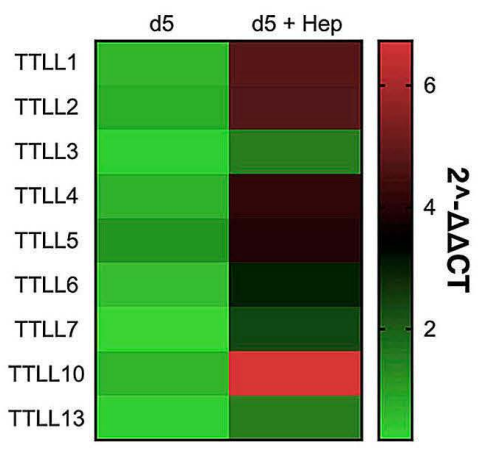

- d5 ・ d5 + Hep

$\mathbf{F}$

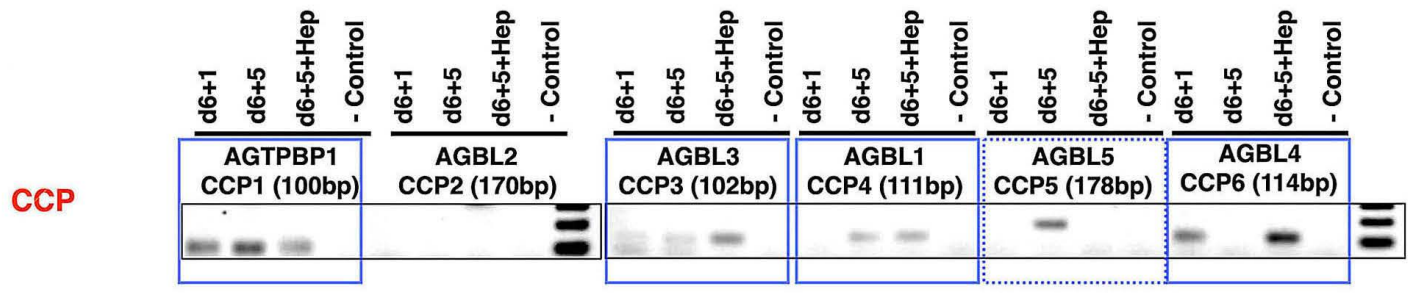

G CCP family change in expression

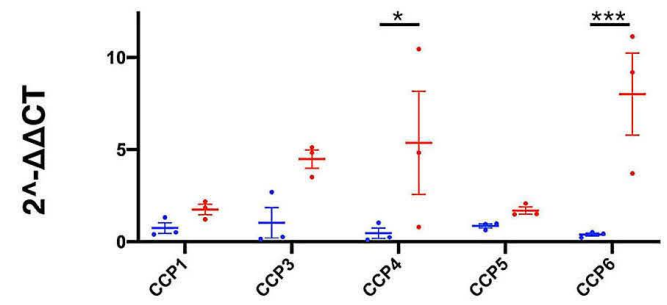

H

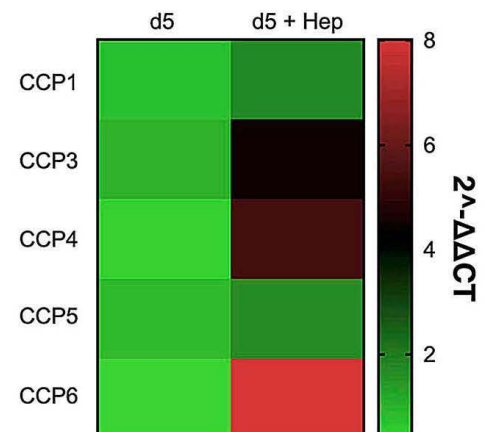




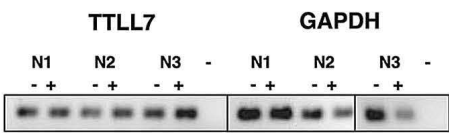

J
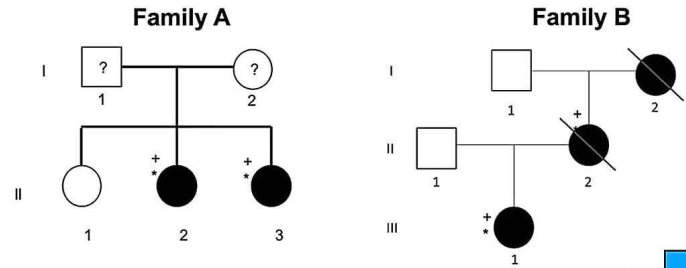

362

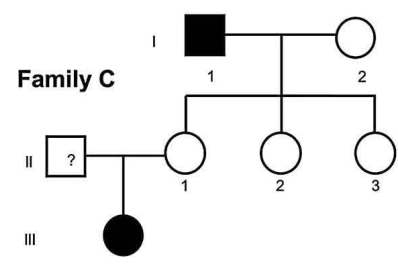

WT
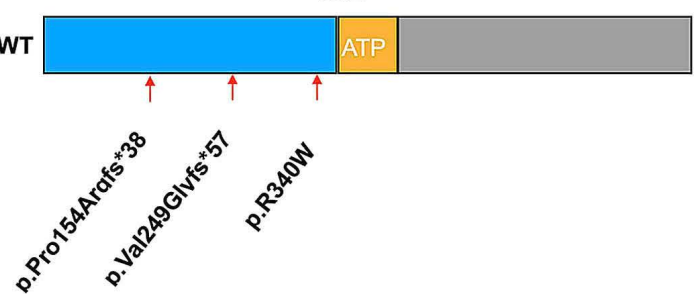

K

\begin{tabular}{|c|c|c|c|c|c|c|c|c|c|}
\hline Individual & A & Sex & $\begin{array}{l}\text { TrLL10 } \\
\text { Variant }\end{array}$ & Protein effect & Platelet count $\left(\mathrm{x} 10^{9} / \mathrm{L}\right)$ & IPF & MPV (fL) & Bleeding Phenotype & Functional Defect \\
\hline A: II:2 & $11(2006)$ & F & c. $462 \mathrm{delG}$ & p.Pro155Argfs*38 & 246 & 4.2 & 11.3 & $\begin{array}{l}\text { Epistaxis, easy bruising and prolonged } \\
\text { bleeding from minor wounds, required }\end{array}$ & Normal aggregometry, secretion. \\
\hline A: II:3 & $10(2008)$ & F & c.462delG & p.Pro155Argfs*38 & 329 & 5.6 & 12.1 & Cutaneous bruising, easy bleeding & Normal aggregometry, secretion. \\
\hline B: III:1 & 29 & M & c.745_746insG & p.Val249Glyfs $* 57$ & $n / a$ & $n / a$ & 9.4 & $\begin{array}{l}\text { Easy bruising, menorrhagia, bled from } \\
\text { tooth extraction. }\end{array}$ & Normal aggregometry, secretion. \\
\hline C: III:1 & 41 & F & c. $1018 \mathrm{C}>\mathrm{T}$ & p.Arg340Trp & $\mathrm{n} / \mathrm{a}$ & $\mathrm{n} / \mathrm{a}$ & 8.0 & $\begin{array}{l}\text { Cutaneous bruising, Menorrhagia, post } \\
\text { partum heemorrhage } \times 2 \text {, bled following }\end{array}$ & Normal aggregometry, secretion. \\
\hline
\end{tabular}

$\mathrm{L}$

\section{Widefield}
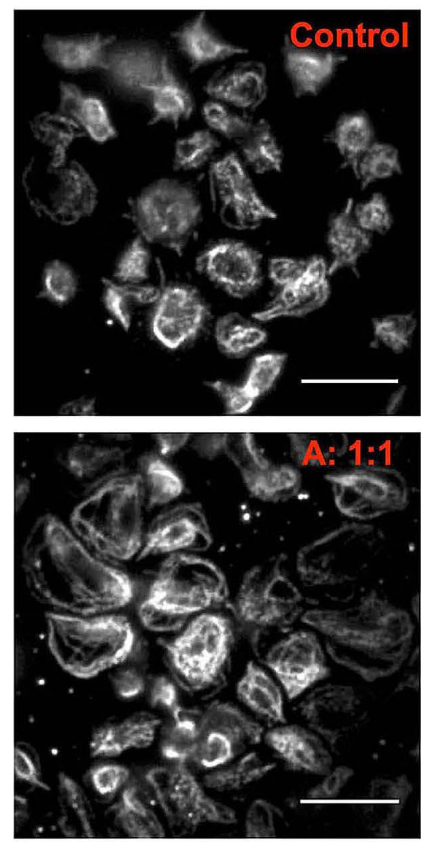

\section{dSTORM}
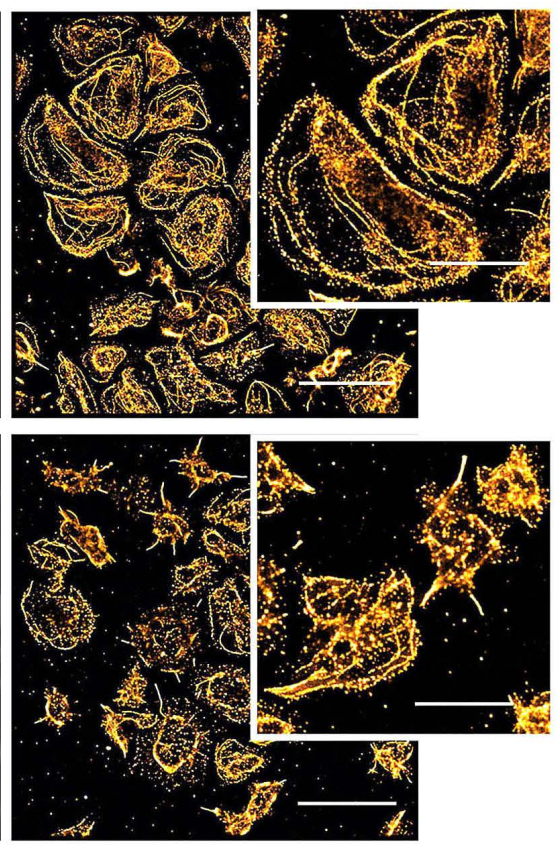

Figure 6. Induced pluripotent stem cell megacaryocytes and platelets differentially express TTLL and CCP enzymes to regulate polymodifications during megakaryocyte maturation and platelet production. The loss of tubulin tyrosine ligase like (TTL) TLL10 is possibly linked to a bleeding phenotype in unrelated human patients. (A) RNA was generated from induced pluripotent stem cell megacaryocytes (iPSC-MK) at different stages of terminal differentiation. Day 1 (d1) day 5 (d5) day 5 cells treated with heparin to induce proplatelet formation ( $\mathrm{d} 5+\mathrm{Hep}$ ) were used to determine whether an upregulation of these enzymes is evident on platelet production. (B) Samples were amplified with housekeeping GAPDH primers (C) A number of TTLL family enzymes were observed, including TTLL1, 2, 3, 4, 5, 6, 7, 10, and 13. Of these, a number appeared to be upregulated in mature and proplatelet forming cells. (D and E) These samples were taken forward and expression was quantified over multiple differentiations using the $\Delta \Delta C T$ method TTLL1, 2, 4, and 10 expression was found to be significantly upregulated on treatment with heparin $(* * \mathrm{P}=$ $0.0081, * P=0.0105, * P=0.0260, * * * P=0.0004$ respectively). ( $F$ to H) A similar panel was performed on cytosolic carboxypeptidase (CCP) enzymes which reverse polymodifications, with expression of CCP1, 3, 4, 5, and 6 was observed. Statistically significant upregulation of CCP4, and ССР 6 were observed on proplatelet production ( $* P=0.0130, * * * P=0.0009$ ). (I) In resting $(-)$ and Creactive protein (CRP) activated $(+)$ platelets from three healthy donors TTLL7 was the only modifying enzyme found to be consistently expressed. (J) Three unrelated families were identified within the Genotyping and Phenotyping of Platelets Study (GAPP) cohort, two with frameshift truncations and one with a missense substitution (p.Pro15Argfs*38, p.Val249Glyfs*57, and p.Arg340Trp respectively, and as shown on the protein schematic of TTLL10). (K) All three families present with normal platelet counts and function, but demonstrate an elevated mean platelet volume (MPV) and consistent histories of bleeding including cutaneous bruising and menorrhagia. $(L)$ Patient $A: 1: 1$ was recalled and demonstrated abnormally large platelets on spreading on fibrinogen when imaged by widefield and single molecule localisation microscopy (tubulin staining, $10 \mu \mathrm{m}$ scale bar, $5 \mu \mathrm{m}$ in cropped image). ( $n=3$, standard deviation. Two-Way ANOVA with multiple comparisons performed. Complete unedited gels found in the Online Supplementary Figures S9 and S10). 


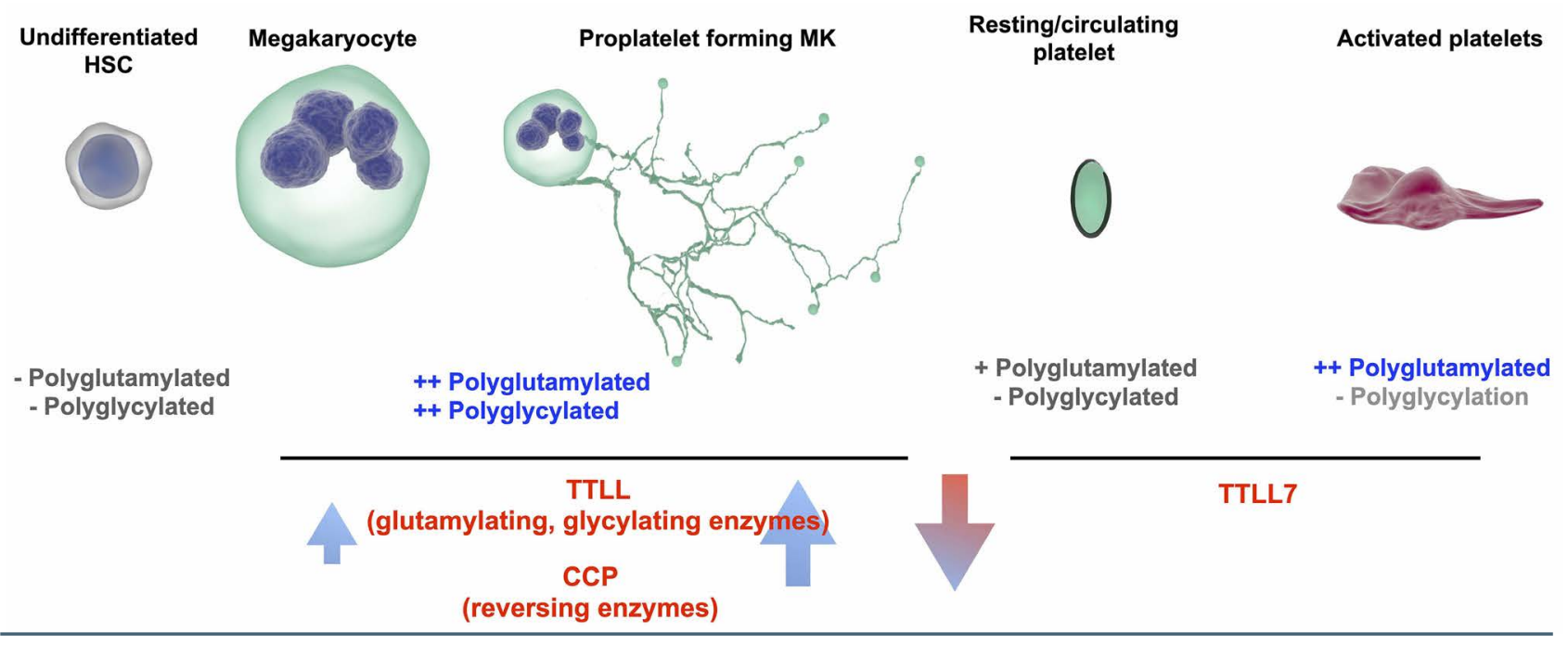

Figure 7. A system of competitive polymodification of TUBB1 driven by the expression of TTLL and CCP enzymes is required for platelet production and function. We observe a system by which as induced pluripotent stem cell megacaryocytes (iPSC-MK) mature and express TUBB1, they acquire both polyglutamylated and polyglycylated tubulin which co-incides with an increase in the expression of glutamylating and glycylating tubulin tyrosine ligase like (TTL) enzymes and reversing cytosolic carboxypeptidase (CCP) enzymes. A resting platelet is partially polyglutamylated, and on activation the marginal band is further polyglutamylated to drive shape change and spreading through the action of TTLL7. We show that this polymodification spatially affects the position of key motor proteins.

\section{TTLL10 variants maybe associated with moderate to severe bleeding in three unrelated families}

Our qRT-PCR screen revealed that a number of TTLL and $\mathrm{CCP}$ are upregulated during the process of platelet production, including the polyglycylase TTLL10. Whole exome sequencing data from patients recruited to the GAPP study identified three unrelated families with rare and novel variants in the TTLL10 gene (Figure 6; Online Supplementary Figures S12 and S13). Extensive in silico analysis was undertaken on the TTLL10 variants which were predicted as 'uncertain significance' using current ACMG guidelines (Online Supplementary Figures S12 and S13). ${ }^{32}$ Two of the three variants result in frameshifts towards the $\mathrm{N}$-terminus of the protein, preceding the ATP binding region (p.Pro15Argfs*38 [novel] and p.Val249Glyfs*57 [frequency $\left.2.15 \times 10^{3}\right]$ ) (Figure $6 \mathrm{~J}$ ). The final family has a missense p.Arg340Trp variant $\left(3.34 \times 10^{* 5}\right.$ frequency).

All three families report normal platelet counts, aggregation and secretion, and present with an established history of moderate to severe bleeding, including cutaneous bruising and menorrhagia (Figure 6K). Family A demonstrated a consistently high MPV (normal ranges MPF [EL] (7.83-10.5), while families $B$ and $C$ do not. Interestingly, one of the patients (A 1:1) was re-recruited, and on platelet spreading on fibrinogen coated coverslips we observed a marked increase in platelet area compared to controls when imaged using widefield and single molecule localisation microscopy (SMLM) (Figure 6L).

\section{Discussion}

We hypothesized that a system of polymodification (polyglutamylation and polyglycylation) targeting the glutamate rich C-terminus of $\beta 1$-tubulin isoform and analogous to similar PTM demonstrated in cilia and neuronal cells, is a likely mechanism by which the interactions of $\beta 1$-tubulin with key motors are regulated in both $\mathrm{MK}$ and platelets.

We report that mature and proplatelet forming CD $42 \mathrm{~b}+$ iPSC-MK demonstrate both polyglutamylation and polyglycylation (polymodification). We observe a markedly dif- ferent distribution of these PTM in the resting platelet, where polyglycylation is lost and polyglutamylation is partially co-localized to the marginal band. On platelet activation, we observe a marked change in the localization of polyglutamylation specific to the marginal band. In iPSCMK with a CRISPR KO of TUBB1, we see a complete loss of proplatelet formation and lose the distinct reorganization of polyglutamylated and polyglycylated tubulin around the periphery of $\mathrm{MK}$ as seen in WT cells.

MK proplatelet extensions are known to be driven by a system of dynein mediated microtubule sliding, while the marginal band in a resting platelet has been shown to be maintained by the antagonistic movement of dynein and kinesin. ${ }^{13,14,36}$ Interestingly, polyglutamylation has been reported as a mechanism of altering motor protein processivity, with in vitro assays suggesting that polyglutamylation of $\beta 1$ tubulin isoforms like TUBB1 and TUBB3 accelerates these motors. ${ }^{28}$ We show a significant effect of polyglutamylation on the spatial localization of dynein and kinesin, supporting in vitro assays which suggest that polyglutamylation is an accelerator of motor proteins.

We report two unrelated patient families with rare TUBB1 C-terminal variants (an R359W missense and a L361Afs*19 frameshift linked to macrothrombocytopenia). Interestingly within family $\mathrm{A}$, a second genetic variant in the $G F I 1 B$ gene, which directly affects the TUBB1 promoter is observed. Individuals within family A positive for the GFI1B variant but WT for TUBB1 have normal platelet counts and a milder increase in MPV and IPF, indicating that the macrothrombocytopenia within the family is possibly linked to the TUBB1 variant, but suggesting a potential additive role for the GFIB $B$ variant which is a focus for future study. Both the clinical phenotype and laboratory investigations points to variable expressivity of the two genetic variants contributing to the overall phenotypes observed.

We go on to show through the expression of these variants in Hek293T cells that each variant results in a dysfunctional $\beta 1$-tubulin protein which is most likely to mirror the effects of a C-terminal truncation.

The system of polymodification evidenced in iPSC-MK 
is analogous to the PTM of ciliated cells, and so we reasoned that axonemal dynein, an isoform of the motor exclusive to axonemes, may play a role in both platelet formation and activation. ${ }^{6,37,38} \mathrm{We}$ find evidence of axonemal dynein on both proplatelet extensions and at the leading edge of spreading platelets. To our knowledge this is the first evidence of a functional role of axonemal dynein outside of classical ciliated structures. In our TUBB1 KO MK, we observe a decrease in the co-localization of dynein to polyglutamyulated tubulin, suggesting that the loss of proplatelet formation observed in these cells is due to a dysregulation of the dynein-mediated microtubule sliding known to drive the elongation of the proplatelet shaft. ${ }^{39}$ The similarities between flagella and MK proplatelet extensions have been discussed previously, Italiano et al. observe structures similar to flagella in taxol treated MK, an idea which led to the microtubule telescoping experiment reported by Patel et al. ${ }^{39,40}$

Our data suggests a tightly regulated, reversible system of polymodification which must be mediated by the cell specific expression of TTLL and CCP. Our expression profiles show a number of TTLL and CCP are expressed by MK, while only the polyglutamylase TTLL7 is expressed by platelets consistent with the different patterns of polymodification oberved in these cells. In MK we find expression of two TTLLs known to be involved in glycylation - the initiase TTLL3 and the elongase TTLL10, with a significant increase in the expression of TTLL10 on platelet production. Our findings support a role of TTLL10 as a polyglycylase on co-expression with TTLL3 as reported by Ikegami et al. in cell lines through co-transfection experiments (Figure 7).

Finally, we report a novel gene, TTLL10, in three unrelated families with excessive bleeding. We identify three unrelated families with TTLL10 variants which result in an increase in an established history of bleeding which provides an invaluable insight to the potential role of polyglycylation in the context of platelet production and function. Our data shows that both TTLL3 and TTLL10 are expressed in platelet producing MK. Our patient cohort do not lose TTLL3 function, and as such the action of TTLL3 as an initiase will occupy glutamate residues which would otherwise be polyglutamylated. In these patients we likely see a loss of polyglycylation, but no coincident increase in polyglutamylation due to the normal function of the initiase (TTLL3). As polyglutamylation and monoglycylation are unaffected, platelet counts (and production) are normal, however, affected individuals appear to have an increased platelet volume and bleeding, suggesting a role for the extended glycine tail in regulating platelet size, with a downstream effect on the ability of platelets to prevent bleeding.
Patel et al. describe a system by which continuous polymerization is key to the dynein-mediated sliding required for platelet production. ${ }^{39}$ This work, alongside reports of tyrosination, acetylation, and polyglutamylation in different MK models, suggests that tubulin PTM are likely part of a highly dynamic system in which polymerizing microtubules are subject to modifications which coordinate platelet production and packaging. Future work will interrogate the interplay between different PTM, and how they fit into a highly dynamic landscape of MT polymerization and function. Indeed, in our KO TUBB1 cells we do not observe a complete loss of polymodification, suggesting that compensatory mechanisms which target other isoforms of tubulin may come into play. There is likely a more extensive effect on the expression of tubulins and their interplay with other cytoskeletal proteins which should be a focus of future work. Similarly, the role of these PTMs will need to be further validated in primary human CD34-derived MK.

This work supports the paradigm of a 'tubulin code' and the importance of microtubule patterning in healthy, and thus diseased, $\mathrm{MK}$ and platelets. We provide novel insights into the mechanisms by which $\beta 1$-tubulin functions in these unique cells.

\section{Disclosures}

No conflicts of interest to disclose.

\section{Contributions}

$A O K$ and NVM devised and performed experiments; $A O K$ and NVM wrote the manuscript; AS performed homology modeling, cloning, transfection, and platelet spreading experiments; $A M$ performed platelet spreading and analysis of patient sequencing data; PLRN performed platelet preparations and reviewed the manuscript; JAP developed and applied image analysis workflows; JSR assisted with western blotting; JY contributed to platelet preparations; all authors reviewed the manuscript.

\section{Acknowledgments}

We thank the families for providing samples and our clinical and laboratory colleagues for their help. The authors would like to thank the TechHub and COMPARE Core facilities at the University of Birmingham. AOK is a Wellcome funded Sir Henry Wellcome Fellow (218649/Z/19/Z). We thank Professor Steve Watson for his ongoing support and invaluable mentorship. The authors do not have any conflict of interest.

\section{Funding}

This work was supported by the British Heart Foundation (PG/13/36/30275; FS/13/70/30521; FS/15/18/31317; $P G / 16 / 103 / 32650 ; I G / 18 / 2 / 33544)$. This research was funded in whole or in part by the Wellcome Trust [218649/Z/19/Z].

\section{References}

1. Wade RH. On and around microtubules: an overview. Mol Biotechnol. 2009;43(2):177191.

2. Gadadhar S, Dadi H, Bodakuntla S, et al. Tubulin glycylation controls primary cilia length. J Cell Biol. 2017; 216(9):2701-2713.

3. Ludueña RF. A hypothesis on the origin and evolution of tubulin. Int Rev Cell Mol Biol. 2013;302:41-185

4. Magiera Maria M, Singh Puja, Janke Carsten.
SnapShot: functions of tubulin posttranslational modifications. Cell. 2018;173(6):1552 1552.e1.

5. Gadadhar S, Bodakuntla S, Natarajan K, Janke C. The tubulin code at a glance. J Cell Sci. 2017;130(8):1347-1353.

6. Wloga D, Joachimiak E, Louka P, Gaertig J Posttranslational modifications of tubulin and cilia. Cold Spring Harb Perspect Biol. 2017;9(6):a028159.

7. Reiter JF, Leroux MR. Genes and molecular pathways underpinning ciliopathies. Nat Rev Mol Cell Biol. 2017;18(9):533-547.
8. Bosch Grau Montserrat, Masson Christel Gadadhar Sudarshan, et al. Alterations in the balance of tubulin glycylation and glutamylation in photoreceptors leads to retina degeneration. J Cell Sci. 2017;130(5):938949.

9. Ikegami K, Sato S, Nakamura K, Ostrowski LE, Setou M. Tubulin polyglutamylation is essential for airway ciliary function through the regulation of beating asymmetry. Proc Natl Acad Sci U S A. 2010;107(23):1049010495.

10. Wu HY, Wei P, Morgan JI. Role of cytosolic 
carboxypeptidase 5 in neuronal survival and spermatogenesis. Sci Rep. 2017;7:41428.

11. Vogel P, Hansen G, Fontenot G, Read R. Tubulin tyrosine ligase-like 1 deficiency results in chronic rhinosinusitis and abnormal development of spermatid flagella in mice. Vet Pathol. 2010;47(4):703-712.

12. Dmitrieff S, Alsina A, Mathur A, Nédélec FJ. Balance of microtubule stiffness and cortical tension determines the size of blood cells with marginal band across species. Proc Natl Acad Sci U S A. 2017;114(17):4418-4423.

13. Diagouraga B, Grichine A, Fertin A, Wang J, Khochbin S, Sadoul K. Motor-driven marginal band coiling promotes cell shape change during platelet activation. J Cell Biol. 2014;204(2):177-185.

14. Sadoul K. New explanations for old observations: marginal band coiling during platelet activation. J Thromb Haemost. 2015;13 (3):333-346.

15. Poulter Natalie S, Thomas SG. Cytoskeletal regulation of platelet formation: coordination of $\mathrm{F}$-actin and microtubules. Int $\mathrm{J}$ Biochem Cell Biol. 2015;66:69-74.

16. Machlus KR, Italiano JE. The incredible journey: from megakaryocyte development to platelet formation. J Cell Biol. 2013;201(6): 785-796.

17. Schwer HD, Lecine P, Tiwari S, Italiano JE, Hartwig JH, Shivdasani RA. A lineagerestricted and divergent beta-tubulin isoform is essential for the biogenesis, structure and function of blood platelets. Curr Biol. 2001;11(8):579-586.

18. Kunishima S, Kobayashi R, Itoh Tomohiko J, Hamaguchi Mo, Saito H. Mutation of the beta1-tubulin gene associated with congenital macrothrombocytopenia affecting microtubule assembly. Blood. 2009;113(2):458461.

19. Kunishima S, Nishimura S, Suzuki H, Imaizumi M, Saito H. TUBB1 mutation disrupting microtubule assembly impairs proplatelet formation and results in congenital macrothrombocytopenia. Eur J Haematol. 2014:92(4):276-282.

20. Fiore M, Goulas C, Pillois X. A new mutation in TUBB1 associated with thrombocytopenia confirms that C-terminal part of b1tubulin plays a role in microtubule assembly. Clin Genet. 2017;91(6):924-926.

21. Natarajan K, Gadadhar S, Souphron J, Magiera MM, Janke Carsten. Molecular interactions between tubulin tails and glutamylases reveal determinants of glutamylation patterns. EMBO Rep. 2017;18(6):1013-1026.

22. Dijk J, Bompard G, Cau J, et al. Microtubule polyglutamylation and acetylation drive microtubule dynamics critical for platelet formation. BMC Biol. 2018;16(1):116.

23. Feng $Q$, Shabrani N, Thon JN, et al. Scalable generation of universal platelets from human induced pluripotent stem cells. Stem Cell Rep. 2014;3(5):817-831.

24. Waterhouse Andrew, Bertoni Martino, Bienert Stefan, et al. SWISS-MODEL homology modelling of protein structures and complexes. Nucleic Acids Res. 2018;46(W1):W296-W303.

25. Bienert S, Waterhouse A, Beer TAP, et al. The SWISS-MODEL Repository-new features and functionality. Nucleic Acids Res. 2016;45(D1):D313-D319.

26. Guex N, Peitsch MC, Schwede T. Automated comparative protein structure modeling with SWISS-MODEL and SwissPdbViewer: a historical perspective. Electrophoresis. 2009;30:S162-S173

27. Benkert P, Biasini M, Schwede T. Toward the estimation of the absolute quality of individual protein structure models. Bioinformatics. 2010;27(3):343-350.

28. Ti SC, Pamula MC, Howes SC, et al. Mutations in human tubulin proximal to the kinesin-binding site alter dynamic instability at microtubule plus- and minus-ends. Dev Cell. 2016;37(1):72-84.

29. Almazni I, Stapley RJ, Khan AO, Morgan NV. A comprehensive bioinformatic analysis of 126 patients with an inherited platelet disorder to identify both sequence and copy number genetic variants. Hum Mutat. 2020;41(11):1848-1865.

30. Johnson B, Lowe GC, Futterer J, et al. Whole exome sequencing identifies genetic variants in inherited thrombocytopenia with secondary qualitative function defects. Haematologica. 2016;101(10):1170-1179.

31. Khan AO, Stapley R, Pike JA, et al. Novel gene variants in patients with platelet-based bleeding using combined exome sequencing and RNAseq murine expression data. J Thromb Haemost. 2021:19(1):262-268.

32. Richards S, Aziz N, Bale S, et al. Standards and guidelines for the interpretation of sequence variants: a joint consensus recommendation of the American College of Medical Genetics and Genomics and the Association for Molecular Pathology. Genet Med. 2015;17(5):405-424

33. Bender M, Thon JN, Ehrlicher AJ, et al. Microtubule sliding drives proplatelet elongation and is dependent on cytoplasmic dynein. Blood. 2015;125(5):860-868

34. Adam F, Kauskot A, Kurowska M, et al. Kinesin-1 Is a new actor involved in platelet secretion and thrombus stability. Arterioscler Thromb Vasc Biol. 2018;38(5): 1037-1051.

35. Ikegami K, Mukai M, Tsuchida J, Heier RL, Macgregor GR, Setou M. TTLL7 is a mammalian beta-tubulin polyglutamylase required for growth of MAP2-positive neurites. J Biol Chem. 2006;281(41):30707-30716. 36. Sirajuddin M, Rice LM, Vale RD. Regulation of microtubule motors by tubulin isotypes and post-translational modifications. Nat Cell Biol. 2014;16(4):335-344.

37. O'Hagan R, Silva M, Nguyen Ken CQ, et al Glutamylation regulates transport, specializes function, and sculpts the structure of cilia. Curr Biol. 2017;27(22):3430-3441.

38. Wloga D, Dave D, Meagley J, Rogowski K, Jerka-Dziadosz M, Gaertig J. Hyperglutamylation of tubulin can either stabilize or destabilize microtubules in the same cell. Eukaryot Cell. 2010;9(1):184-193.

39. Patel R, Richardson Jennifer L, Schulze Harald, et al. Differential roles of microtubule assembly and sliding in proplatelet formation by megakaryocytes. Blood. 2005;106(13):4076-4085.

40. Italiano JE, Lecine P, Shivdasani R A Hartwig J H. Blood platelets are assembled principally at the ends of proplatelet processes produced by differentiated megakaryocytes. J Cell Biol. 1999;147(6) 1299-1312.

41. Kremers G, Hazelwood KL, Murphy CS, Davidson MW, Piston DW. Photoconversion in orange and red fluorescent proteins. Nat Methods. 2009;6(5):355358.

42. Khan AO, Simms VA, Pike JA, Thomas SG Morgan NV. CRISPR-Cas9 mediated labelling allows for single molecule imaging and resolution. Sci Rep. 2017;7(1):8450.

43. Khan AO, White CW, Pike JA, et al Optimised insert design for improved singlemolecule imaging and quantification through CRISPR-Cas9 mediated knock-in Sci Rep. 2019;9(1):14219.

44. Smith CW, Raslan Z, Parfitt L, et al. TREM like transcript 1: a more sensitive marker of platelet activation than P-selectin in humans and mice. Blood Adv. 2018:2(16):2072-2078.

45. Pike JA, Simms VA, Smith CW, et al. An adaptable analysis workflow for characterization of platelet spreading and morphology. Platelets. 2020:1-5

46. Berthold MR, Cebron N, Dill F, et al KNIME-the konstanz information miner: version 2.0 and beyond. AcM SIGKDD explorations Newsletter. 2009;11:26-31.

47. Sommer C, Strahle C, Kothe F, A. Hamptrecht F. ilastik: Interactive Learning and Segmentation Toolkit. In: Eighth IEEE International Symposium on Biomedical Imaging (ISBI). Proceedings. 2011;230-233.

48. Khan AO, Maclachlan A, Lowe GC, et al. High-throughput platelet spreading analysis: a tool for the diagnosis of platelet-based bleeding disorders. Haematologica. 2020;105(3):e124-e128.

49. McDonald JH, Dunn KW. Statistical tests for measures of colocalization in biological microscopy. J Microsc. 2013;252(3):295302. 